\title{
Edad y origen de la paleocuenca continental de Teocaltiche, México
}

\section{Norma González-Cervantes ${ }^{1,3}$, José Jorge Aranda-Gómez ${ }^{2, *}$, Oscar Carranza-Castañeda ${ }^{2}$ y Carlos Ortega-Obregón ${ }^{2}$}

\author{
${ }^{1}$ Posgrado en Ciencias de la Tierra, Universidad Nacional Autónoma de México, \\ Boulevard Juriquilla 3001, 76230 Querétaro, Qro., México. \\ ${ }^{2}$ Centro de Geociencias, Universidad Nacional Autónoma de México, \\ Boulevard Juriquilla 3001, 76230 Querétaro, Qro., México. \\ ${ }^{3}$ Departamento de Ingeniería Civil, Universidad Autónoma de Aguascalientes, \\ Avenida Universidad 940, Aguascalientes, Ags., 20131 México. \\ *jjag@geociencias.unam.mx
}

\section{RESUMEN}

En la región ubicada al este de la parte sudoriental de la Sierra Madre Occidental (SMOc) existió un lago extenso durante parte del Oligoceno y casi todo el Mioceno. El lago se formó en una depresión tectónica, asociada con el graben de Aguascalientes y el semigraben de Teocaltiche. Los sedimentos que rellenaron parcialmente esta cuenca continental incluyen sedimentos epiclásticos derivados de las rocas de la SMOc y de la Mesa Central, precipitados químicos y bioquímicos (caliza de agua dulce) y sedimentos mixtos, así como una proporción pequeña, pero generalizada, de capas de ceniza volcánica de caída, que fueron empleadas para obtener edades de U-Pb en circón. El alcance temporal del relleno de la cuenca es amplio, pues abarca desde el Rupeliano ( 28.6 Ma) hasta el Tortoniano ( 7.6 Ma). Previamente, con base en fauna fósil se creía que la sucesión era solo del Mioceno tardío.

En la parte sur de la SMOc hay varias cuencas de origen tectónico, con orientación NNE, que contuvieron lagos. En al menos tres de ellas (Tlaltenango, Juchipila y Teocaltiche) se sabe, por el carácter de los sedimentos y por sus fósiles del Henfiliano temprano, que hubo lagos de manera simultánea. En el graben de Bolaños, al oeste y paralelo a la cuenca de Tlaltenango, se ha propuesto la existencia de un lago durante el Neógeno, cuyos sedimentos están casi completamente erosionados, por lo que se sabe poco acerca de ellos. Para explicar el bloqueo parcial y simultáneo de los sistemas de drenaje se requiere de una causa común de carácter regional. Las cuatro cuencas tectónicas mencionadas terminan al norte de las depresiones asociadas a los rifts Tepic-Zacoalco o Tula-Chapala. El ajuste isostático en los bloques de piso de estos rifts pudo causar el cierre parcial de los ríos axiales en los grábenes con rumbos NNE y atrapar así los sedimentos. Aunque se desconoce con precisión cuando las cuencas dejaron de ser sistemas acumulativos, se cree, por la ausencia de fósiles del Blancano, que la apertura pudo suceder antes de $\sim 4.75 \mathrm{Ma}$, valor numérico del inicio de esa etapa faunística. Una causa común probable de la apertura puede ser la invasión marina de una depresión en lo que actualmente es el Golfo de California. La creación de este nuevo nivel base de erosión pudo aumentar considerablemente el gradiente hidráulico en los sistemas fluviales, proveyendo la energía suficiente para remontar al levantamiento asociado a la actividad de los rifts.

Palabras clave: paleolago; secuencia volcánica-sedimentaria; edades U-Pb en circón; paleocuenca Teocaltiche; semigraben de Teocaltiche; graben de Aguascalientes; México.

\section{ABSTRACT}

An extensive lake existed during part of the Oligocene and almost all the Miocene in the region located east of the southern end of the Sierra Madre Occidental (SMOc). The lake was formed in a tectonic depression associated with the Aguascalientes graben and the Teocaltiche half-graben. Sediments accumulated inside this continental basin are epiclastic, mainly derived from volcanic rocks of the SMOc, and from the volcanic cover of the Mesa Central, chemical and biochemical precipitates (fresh water limestone), and mixed sediments, as well as far less voluminous, but ubiquitous, volcanic ash-fall layers, which were used to obtain $U-P b$ ages from zircons. The age range of the basin-fill sediments is broad, varying from Rupelian ( 28.6 Ma) to Tortonian $(\sim 7.6 \mathrm{Ma})$. Previously, on the basis of fossil fauna, the succession was considered only as late Miocene in age.

The southern end of the SMOc includes several NNE-trending basins of tectonic origin that contained lakes. Based on the characteristics of the individual sedimentary successions, and on their fossil faunas, it is known that three of these basins (Tlaltenango, Juchipila and Teocaltiche) had lakes at the same time. The fourth basin, the Bolaños graben, located west and parallel to the Tlaltenango basin, probably also had a lake during the Neogene, but almost all its sediments have been eroded, so that little is known about them. In order to explain the simultaneous partial closure of all the drainage systems a common, regional, cause must be invoked. All the tectonic basins in the southern part of the SMOc end close to the tectonic depressions related to either the Tepic-Zacoalco or Tula-Chapala rifts. Isostatic uplift of the footwall blocks of these $\sim E$-W-trending rifts 
may have provoked partial closure of hydrologic systems within the NNE-trending grabens. It is not known when the basins were opened and ceased to act as aggrading systems, but the apparent absence of Blancan age fossils within them may indicate that they could have been opened prior to $\sim 4.75 \mathrm{Ma}$, which is the numerical value of the beginning of this faunal stage. A probable common cause for the simultaneous opening could be a marine invasion in the region now occupied by the Gulf of California. The new base level of erosion probably increased the stream gradients in the fluvial systems, providing enough energy to the axial rivers within the grabens to overcome the uplift associated with $\sim E-W$ rift activity.

Key words: paleo-lake; volcanic-sedimentary sequence; zircon $\mathrm{U}-\mathrm{Pb}$ ages; Teocaltiche paleo-basin; Teocaltiche half-graben; Aguascalientes graben; Mexico.

\section{INTRODUCCIÓN}

En una amplia región del centro de México es común encontrar afloramientos extensos de sedimentos cenozoicos de origen fluviolacustre. El área estudiada en este trabajo (Figura 1) corresponde al relleno sedimentario acumulado durante el Cenozoico tardío en las partes bajas del semigraben de Teocaltiche, localizado en la porción sudeste de la Sierra Madre Occidental (SMOc). Una parte de estos sedimentos, expuestos en al área cercana a Yahualica, Jalisco (Figura 1b) fue investigada por Caravella (1985), quien propuso la hipótesis del cierre de una cuenca fluvial que fue represada por coladas volcánicas coalescentes, y que estaba limitada al oeste por el pilar tectónico de la sierra de Nochistlán (Figura 1b: SN). De acuerdo con Caravella (1985), la cuenca se extendería desde Yahualica hasta la ciudad de Aguascalientes. Con base en el estudio de los sedimentos expuestos en la carta Yahualica, ese autor reporta que la cuenca estaría parcialmente rellena por (1) depósitos de lodolita y arena fina de ambientes deltáicos, (2) intercalación de lodolita con caliza y con micrita lodosa de ambiente lacustre marginal y (3) caliza micrítica lodosa representativa de ambiente lacustre abierto. Silicificación, en forma de ópalo, es un cambio diagenético común en todos estos sedimentos, a los que él atribuyó una probable edad cuaternaria.

Posteriormente, se propuso la hipótesis de la existencia, a finales del Cenozoico, de una cuenca lacustre muy extensa en el centro de México, a la que se llamó Lago Xalisco (De Cserna y Álvarez, 1995). De acuerdo con esa hipótesis, el lago se extendía desde las inmediaciones de Toluca hasta Guadalajara y alcanzaba los sitios en donde actualmente se ubican las ciudades de León y Aguascalientes (Figura 1a). Aparentemente, ese paleolago se formó durante una época pluvial (Neógeno-Cuaternario)

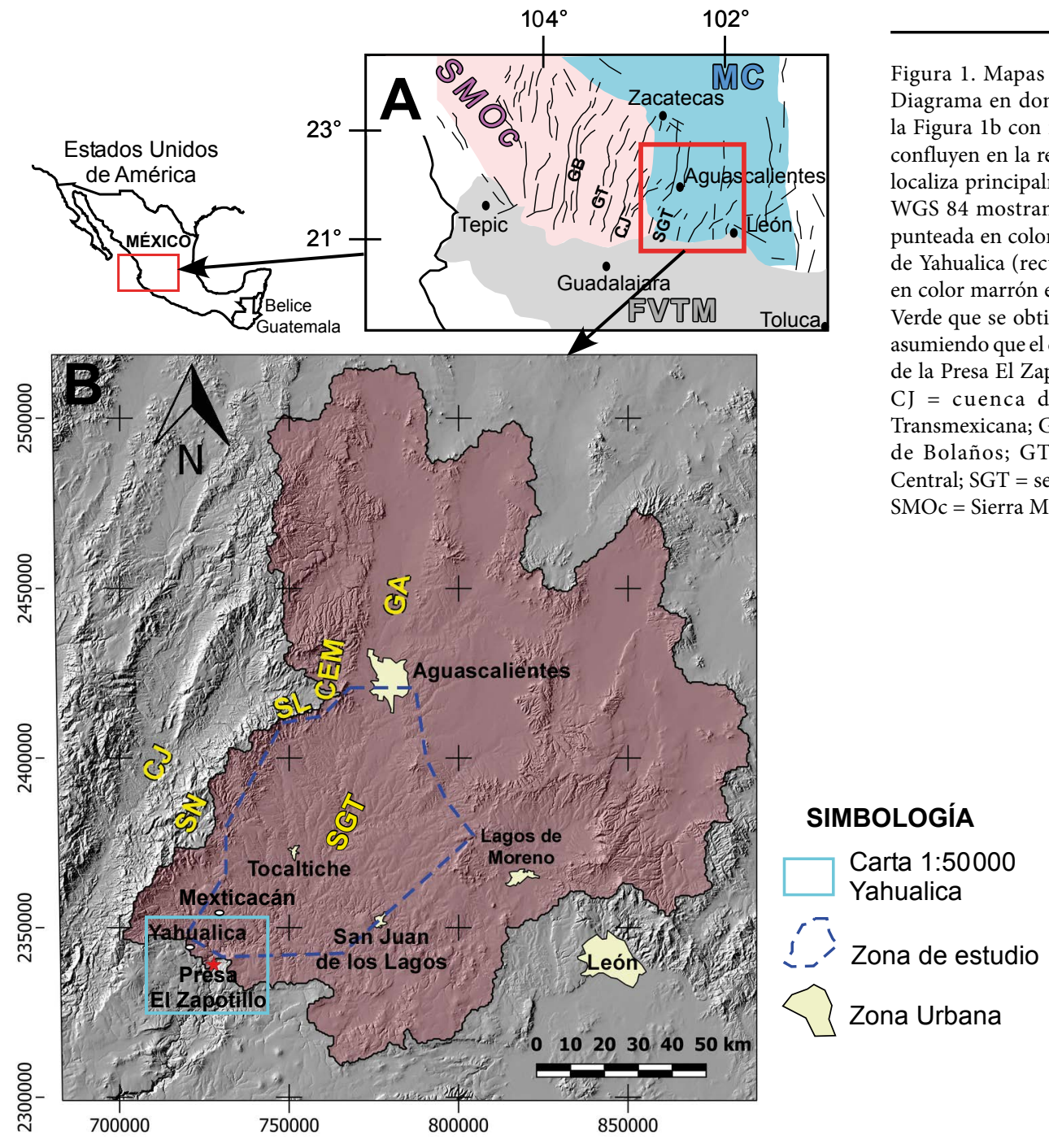


como consecuencia de movimientos geodinámicos que obstruyeron el drenaje del río Lerma ancestral (De Cserna y Álvarez, 1995).

Al occidente del área de estudio se localiza la cuenca de Juchipila (Figura 1b: CJ), donde se ha reconocido una secuencia sedimentaria similar a la estudiada en este trabajo. Estudios de campo de la estratigrafía de algunas secciones (López, 1991, Carranza-Castañeda et al., 2013) y petrografía de muestras sedimentarias colectadas en ellas (Lahiere, 1982 y López, 1991) han permitido identificar diferentes asociaciones de facies de ambientes lacustre, fluvial, de llanura de inundación y de abanico aluvial intercaladas de manera compleja. En ese relleno de graben son comunes las capas de ceniza volcánica intercaladas con otros sedimentos. A esa secuencia sedimentaria se le dio el nombre informal de formación Juchipila (Lahiere, 1982) haciéndose notar que el contacto entre la formación Juchipila y los depósitos volcánicos del Complejo Volcánico Superior de la SMOc es una inconformidad estratigráfica. Fósiles de vertebrados colectados en la formación Juchipila indican que esta incluye sedimentos con edades del Henfiliano temprano ( 6.8 $4.75 \mathrm{Ma})$, mientras que edades de circones recuperados de capas de ceniza volcánica intercaladas con los sedimentos oscilan entre 6.95 y $5.59 \mathrm{Ma}$ (Carranza-Castañeda et al., 2013). En los trabajos de Lahiere (1982) y de López (1991) también se señaló que la distribución vertical de litofacies en la secuencia sedimentaria está influenciada, principalmente, por cambios tectónicos contemporáneos a la sedimentación y, en menor grado, por ciclos climáticos. Lahiere (1982) menciona la existencia de dos familias de fallas importantes (NNE-SSW y E-W) en la cuenca y sus alrededores. Una conclusión importante de López (1991) es que al comparar la fauna de ostrácodos encontrada en sedimentos del paleolago Juchipila con ostrácodos de sistemas lacustres modernos se puede inferir que el paleolago era perenne, de cuenca abierta y se desarrolló en un clima cálido subtropical. Por otro lado, la gran diversidad de mamíferos fósiles hasta ahora colectados en la formación Juchipila es consistente con un ambiente comparable con una sabana abierta (Carranza-Castañeda et al., 2013).

Estableciendo una comparación entre lo que se sabe acerca del sistema fluvio-lacustre de Juchipila y la información colectada por Caravella (1985) en la parte correspondiente a "lago abierto" del sistema de Teocaltiche es posible plantear la hipótesis de que en el área de Teocaltiche haya habido una evolución sedimentológica similar a la del paleolago de Juchipila y que estas dos cuencas hayan sido en parte contemporáneas. En este trabajo se expondrán e interpretarán datos obtenidos en los sedimentos de la paleocuenca de Teocaltiche, tanto en campo como en laboratorio, los cuales son principalmente de carácter fisiográfico, litológico y geocronológico. La interpretación temporal de la evolución de la cuenca está respalda por edades $\mathrm{U}-\mathrm{Pb}$ de circones recuperados de muestras de capas de ceniza de caída, una ignimbrita y circones detríticos provenientes de un depósito de arena, así como por la edad paleontológica de un fragmento de la mandíbula de un caballo fósil.

A pesar de que Caravella (1985) se refirió al área de estudio como la "cuenca de Yahualica", en este trabajo se emplearán los nombres de paleocuenca y semigraben de Teocaltiche y paleo-río Verde para referirse a la zona de acumulación de sedimentos, a la estructura que controló la ubicación de la cuenca y al río axial que drenaba al semigraben, respectivamente, ya que la extensión de estos rasgos exceden con mucho al área cubierta por el estudio de dicho autor y porque, estrictamente hablando, el poblado de Yahualica se encuentra en el margen o ligeramente afuera de la cuenca en donde se acumularon los sedimentos (Figura 1b, recuadro en azul claro, carta 1:50000 de Yahualica). La investigación realizada comprende desde la parte central del estado de Aguascalientes (i.e., la parte meridional del graben de Aguascalientes) a la región entre Yahualica y San Juan de los Lagos, en el estado de Jalisco (i.e,. el semigraben de Teocaltiche).

\section{Entorno geológico regional}

En el área de estudio se unen las porciones sudoriental de la SMOc, sudoccidental de la Mesa Central (MC) y noroccidental de la Faja Volcánica Transmexicana (FVTM) (Figura 1a). Actualmente el río principal en la zona referida fluye de norte a sur y recibe distintos nombres, dependiendo de la ubicación geográfica; se le llama San Pedro al norte de la ciudad de Aguascalientes y Verde cerca del límite meridional del área de estudio (Figura 2). En el sitio en donde el río San Pedro cambia su dirección dominante de NNE-SSW a NE-SW se le nombra Agostaderito. Para simplificar, en este trabajo se hará referencia a él como río Verde, que es el nombre que conserva hasta su confluencia con el río Grande de Santiago.

De manera general, la estratigrafía de las rocas cenozoicas del área se dividió en tres grandes sucesiones, que de más antigua a más reciente son: 1) secuencia volcánica de la SMOc, compuesta por riolitas $\geq$ ignimbritas félsicas $>$ andesitas, 2) secuencia sedimentaria-volcánica de la cuenca de Teocaltiche: depósitos fluviales, aluviales, lacustres y eólicos de la paleocuenca Teocaltiche y rocas volcánicas intracuenca, así como cenizas extracuenca intercaladas en estos, y 3) secuencia de la FVTM que en el área está formada principalmente por derrames de lava andesítico-basáltica y por algunas ignimbritas félsicas, posteriores a las de la SMOc.

Las rocas volcánicas de la SMOc en el área de estudio y sus alrededores son dominantemente riolíticas a riodacíticas y varían en edad entre 47 y $26 \mathrm{Ma}$ (Figura 3 y Tabla 1). En el extremo noroccidental del área de estudio (entre la presa Los Arquitos y el cerro El Muerto) Nieto-Samaniego et al. (1996) fecharon (K-Ar, sanidino) rocas volcánicas félsicas con edades $47.2 \pm 1.2 \mathrm{Ma}$ y $40.6 \pm 1.0 \mathrm{Ma}$ (Figura 3, localidades 1 y 2 , respectivamente y Tabla 1 ). Los mismos

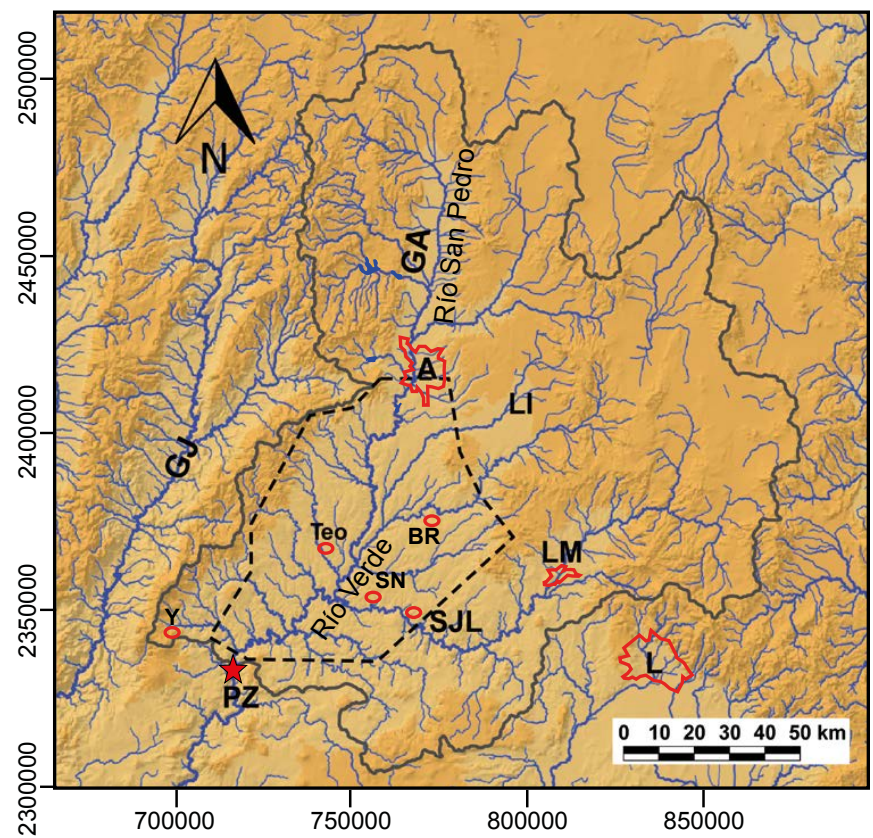

Figura 2. Red fluvial en la parte alta de la cuenca hidrológica del río Verde, generada con el simulador SIATL (INEGI, 2010). El mapa se localiza principalmente en el cuadrante 13Q con un DATUM WGS 84. Nótese que los tributarios en la parte oriental de la cuenca son considerablemente más largos que aquellos en el lado occidental y que el río axial actual se encuentra más cerca del bloque de piso del semigraben de Teocaltiche. Acrónimos: $\mathrm{A}=$ Aguascalientes, $\mathrm{BR}=$ Belén del Refugio, GA = Graben de Aguascalientes, GJ = Graben de Juchipila, L = León, $\mathrm{Ll}=$ El Llano, LM = Lagos de Moreno, PZ = Presa El Zapotillo, SJL = San Juan de los Lagos, $\mathrm{SN}=$ San Nicolás, Teo $=$ Teocaltiche y $\mathrm{Y}=$ Yahualica. 
autores obtuvieron una edad 26.6 $\pm 0.7 \mathrm{Ma}$ (K-Ar, sanidino) para una ignimbrita de la Sierra de Nochistlán (Figura 3, loc. 3 y Tabla 1). Las andesitas basálticas de la parte centro-septentrional de la FVTM en el área tienen edades (K-Ar, roca total) que oscilan entre 10.96 $\pm 0.57 \mathrm{Ma}$ y $10.52 \pm 0.22 \mathrm{Ma}$ (Figura 3, localidades 4 y 5 , respectivamente y Tabla 1 ) en muestras colectadas en las inmediaciones de la Sierra de Nochistlán y que probablemente son parte de rocas transicionales entre la SMOc y la FVTM (Delgado-Argote et al., 1981; Nieto-Obregón et al., 1981). Finalmente, la edad para los basaltos de la FVTM más cercanos al área de estudio es de $8.52 \pm 0.18 \mathrm{Ma}$ (K-Ar, roca total: Nieto-Obregón et al., 1981), estos basaltos están expuestos al sur de Cañadas de Obregón y de la presa El Zapotillo (Figura 3, loc. 6 y Tabla 1).

Para la secuencia sedimentaria-volcánica que conforma el relleno del semigraben de Teocaltiche y de la parte meridional del graben de Aguascalientes hasta ahora no se han reportado edades isotópicas, solo se conocen edades biocronológicas reportadas por diferentes autores (Figura 3): en sedimentos sobrela carretera que va de Teocaltiche a San Juan de los Lagos se han colectado fósiles de hiena, un antilocáprido, équidos y un gonfoterio indeterminado del Henfiliano tardío (6.8 - 4.75 Ma: Montellano-Ballesteros, 1997, Tedford et al., 2004). Otro ejemplar de Osteoborus sp. (hiena) fue colectado cerca de Belén del Refugio por Guzmán-Gutiérrez y Rodríguez-Huerta (1991). Cerca de San Juan de losLagos se han reportado fósiles de aves, un camélido (Lamaichnum macropodum), un proboscidio y huellas de félido del
Neógeno (Rodríguez-de la Rosa y Guzmán-Gutiérrez, 2012), así como huellas de felino del Plioceno superior-Pleistoceno (Dugés, 1894). De igual manera, en Aguascalientes en la localidad conocida como El Cedazo se han colectado fósiles de équidos del Pleistoceno temprano (Mooser,1958; Mooser y Dalquest, 1975; Montellano-Ballesteros, 1989, 1990) en lo que Hernández-Láscares (1981) llamó toba Aguascalientes. Los sedimentos expuestos en El Cedazo son capas de arena y gravacon estratificación cruzada que sobreyacen a una ignimbrita de grado bajo, por lo que se cree que los fósiles provienen de los sedimentos.

En la cuenca de Juchipila, al oeste del área de estudio (Figura 3), Carranza-Castañeda et al. (2006b, 2013), reportaron una secuencia de sedimentos fluvio-lacustres similar a la de Teocaltiche, con una fauna fósil del Henfiliano temprano que incluye Calippus hondurensis. Estos mismos autores señalan que los sedimentos lacustres se encuentran intercalados con cenizas volcánicas con edades U-Pb (circón) de $6.95 \pm 0.27$ a $5.59 \pm 0.11 \mathrm{Ma}$. Por lo tanto, las cuencas sedimentarias continentales de Juchipila y Teocaltiche fueron al menos en parte contemporáneas.

En la literatura, el relleno epiclástico-volcánico (i.e., la secuencia sedimentaria-volcánica) del área de estudio se ha descrito como sedimentos lacustres en la región entre Yahualica, Teocaltiche, San Juan de los Lagos, Encarnación de Díaz y Villa Hidalgo (Dugés, 1894; INEGI, 1971b, 1973a, 1973b, 1973c, 1980; Caravella, 1985; Montellano-Ballesteros, 1997; SGM, 1998; IMTA, 2007; Rodríguez-

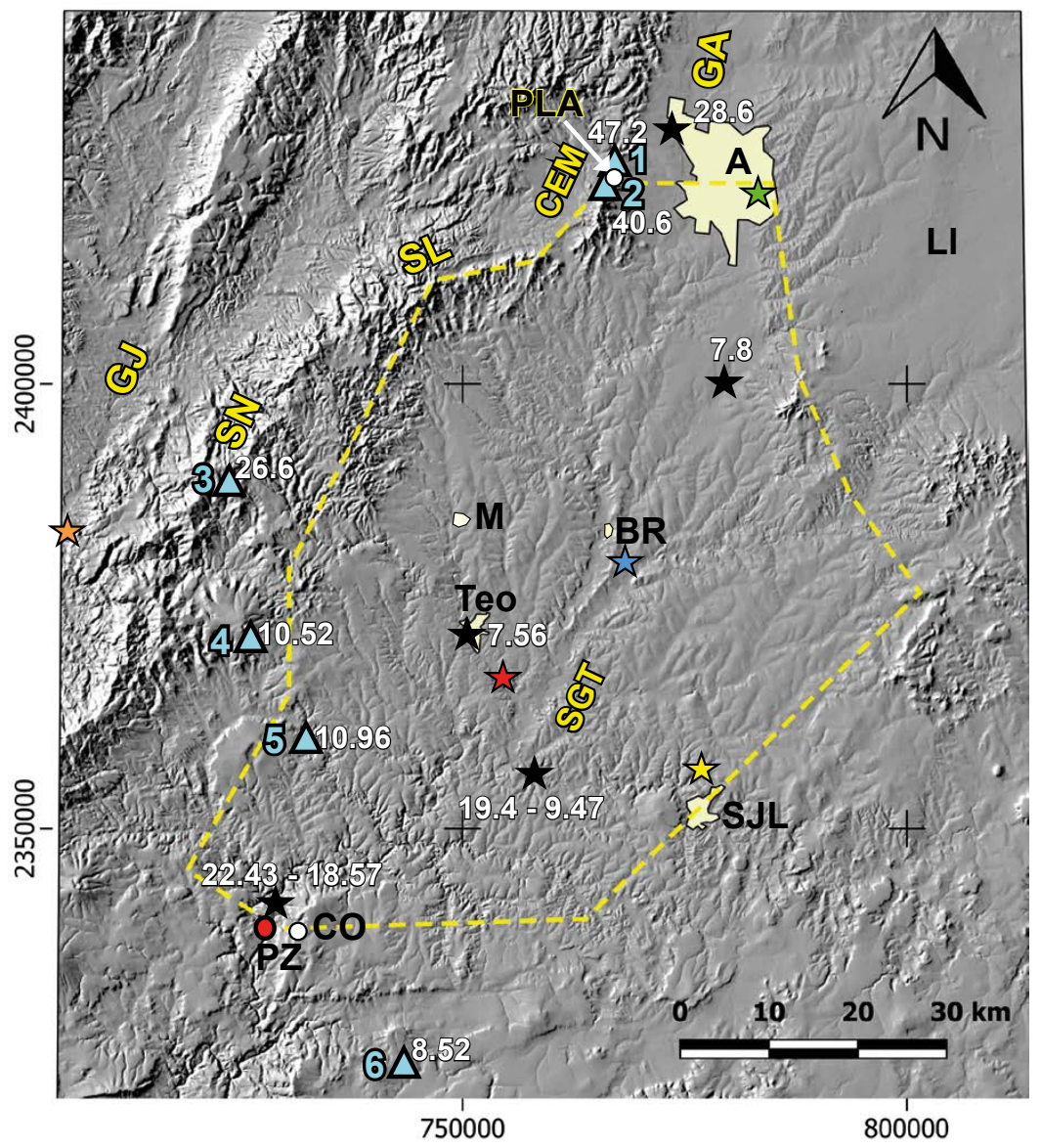

\section{SIMBOLOGÍA}

ESTRUCTURAS GEOLÓGICAS

GJ = Graben de Juchipila

$\mathrm{GA}=$ Graben de Aguascalientes

SGT = Semigraben de Teocaltiche

ESTRUCTURAS MORFOLÓGICAS

$\mathrm{SN}=$ Sierra de Nochistlán

$\mathrm{SL}=$ Sierra Laurel

CEM $=$ Cerro El Muerto

\section{ZONAS URBANAS}

$\mathrm{A}=$ Aguascalientes $\quad B R=$ Belén del Refugio

$\begin{array}{ll}\text { Teo }=\text { Teocaltiche } & B R=\text { Belén del Refugio } \\ \text { SJL }=\text { San Juan de los Lagos } & M=\text { Mechoacanejo }\end{array}$

PZ = Presa "El Zapotillo" PLA = Presa "Los Arquitos"

$\mathrm{LI}=$ EI Llano $\quad \mathrm{CO}=$ Cañadas de Obregón

\section{REGISTROS PALEONTOLÓGICOS}

Wr Équidos del Pleistoceno temprano (Mooser, 1958; Mooser y Dalquest, 1975; Montellano-Ballesteros, 1989, 1990).

Aves, un camélido (Lamaichnum macropodum), un

ک proboscídeo y huellas de félido del Neógeno (Rodríguez-

de la Rosa y Guzmán-Gutiérrez, 2012) huellas de felino del Plioceno superior-Pleistoceno (Dugés, 1894).

政 Osteoborus (hiena) (Guzmán-Gutiérrez y RodríguezHuerta, 1991).

负 $6.8-4.75 \mathrm{Ma}$ : hiena, un antilocáprido, équidos y un gonfoterio (Montellano-Ballesteros, 1997; Tedford et al., 2004).

นૅ 6.53 - 5.59 Ma: Équido Calippus Hondurensis del Henfiliano temprano (Carranza-Castañeda et al., 2013).

\section{EDADES ISOTÓPICAS}

$\triangle$ Datos de la literatura.

Datos de este trabajo; mayor información en el texto.

Nota: La ubicación de algunas edades paleontológicas es aproximada.

Figura 3. Ubicación de las rocas volcánicas fechadas en este trabajo y otras edades reportadas en la literatura. El círculo rojo corresponde a la Presa El Zapotillo, cercano al sitio en donde probablemente se cerró la cuenca en el Mioceno. Las estrellas negras muestran los sitios en donde se colectaron muestras para fechamientos $\mathrm{U}-\mathrm{Pb}$ (este trabajo) y los números rojos son las edades obtenidas en Ma. El mapa se localiza en el cuadrante 13Q con un DATUM WGS 84. Datos de la literatura: puntos 1, 2 y 3 de Nieto-Samaniego et al. (1996); punto 4 de Delgado-Argote et al. (1981); y puntos 5 y 6 de Nieto-Obregón et al. (1981). 
Tabla 1. Edades de las rocas volcánicas reportadas en la literatura para el área de estudio y sus alrededores (véase ubicación en Figura 3).

\begin{tabular}{|c|c|c|c|c|c|c|}
\hline $\begin{array}{l}\text { Ubicación en } \\
\text { Figura } 3\end{array}$ & Localidad & Roca & Método & $\begin{array}{l}\text { Material } \\
\text { fechado }\end{array}$ & $\begin{array}{l}\text { Edad } \\
(\mathrm{Ma})\end{array}$ & Referencia \\
\hline 1 & $\begin{array}{l}\text { Los Arquitos, } \\
\text { Aguascalientes }\end{array}$ & $\begin{array}{l}\text { Roca volcánica } \\
\text { félsica }\end{array}$ & $\mathrm{K}-\mathrm{Ar}$ & Sanidino & $47.2 \pm 1.2$ & Nieto-Samaniego et al., 1996. \\
\hline 2 & $\begin{array}{l}\text { Cerro El Muerto, } \\
\text { Aguascalientes }\end{array}$ & $\begin{array}{l}\text { Roca volcánica } \\
\text { félsica }\end{array}$ & $\mathrm{K}-\mathrm{Ar}$ & Sanidino & $40.6 \pm 1.0$ & Nieto-Samaniego et al., 1996. \\
\hline 3 & Sierra de Nochistlán & Ignimbrita & $\mathrm{K}-\mathrm{Ar}$ & Sanidino & $26.6 \pm 0.7$ & Nieto-Samaniego et al., 1996. \\
\hline 4 & $\begin{array}{l}\text { Límites de la Sierra de } \\
\text { Nochistlán }\end{array}$ & $\begin{array}{l}\text { Andesita- } \\
\text { Basalto }\end{array}$ & $\mathrm{K}-\mathrm{Ar}$ & Roca total & $10.52 \pm 0.22$ & Delgado-Argote et al., 1981. \\
\hline 5 & $\begin{array}{l}\text { Límites de la Sierra de } \\
\text { Nochistlán }\end{array}$ & $\begin{array}{l}\text { Andesita- } \\
\text { Basalto }\end{array}$ & $\mathrm{K}-\mathrm{Ar}$ & Roca total & $10.96 \pm 0.57$ & Nieto-Obregón et al., 1981. \\
\hline 6 & $\begin{array}{l}\text { Cañadas de Obregón y } \\
\text { Presa el Zapotillo }\end{array}$ & $\begin{array}{l}\text { Andesita- } \\
\text { Basalto }\end{array}$ & $\mathrm{K}-\mathrm{Ar}$ & Roca total & $8.52 \pm 0.18$ & Nieto-Obregón et al., 1981. \\
\hline
\end{tabular}

de la Rosa y Guzmán-Gutiérrez, 2012), mientras que los sedimentos del graben de Aguascalientes y de la región conocida como El Llano (Figura 2) se registran como sedimentos fluvio-lacustres intercalados con lavas (SGM, 1998; Aguirre-Díaz y Carranza-Castañeda, 2000, UAQ-UNAM-UAA, 2002). En la descripción de los sedimentos del graben de Aguascalientes y de Teocaltiche comúnmente se hace alusión a un paquete sedimentario continental, relativamente grueso (hasta $200 \mathrm{~m}$ de espesor) de arenisca arcillosa intercalada con capas y/o lentes de conglomerado y con tobas líticas de color blanco que incluyen clastos derivados de rocas volcánicas (SMOc) mezclados con la ceniza en forma caótica (SGM, 1998). Los horizontes de grava o conglomerado contienen clastos en una matriz de arena gruesa a mediana y son cuerpos aislados que se encuentran disgregados y transportados adentro del graben de Aguascalientes, así como arenas y limos (SGM, 1998). A la secuencia de arena fina con estratificación cruzada, con capas de lapilli masiva y de ceniza riolítica se le denomina "toba Aguascalientes" de acuerdo con lo propuesto por HernándezLáscares (1981). En las cartas geológicas 1:50000 del área de estudio el INEGI (1971a, 1971b, 1973a, 1973b, 1973c, 1973d, 1977, 1980 y 1981) cartografió la secuencia sedimentaria-volcánica como cuerpos de caliza, caliza-lutita, marga-lutita, lutita-arenisca, arenisca, areniscaconglomerado, conglomerado, brecha, yeso, travertino y tilita (sic). En esos mapas las litologías de grano grueso predominan hacia la zona norte del área de estudio, mientras que la caliza y sedimentos de grano fino predominan hacia la parte sur.

El trabajo de Caravella (1985) se centró en un área (véase ubicación de la Hoja Yahualica en Figura 1b) en donde predominan los depósitos de ambiente deltáico, lacustre marginal y lacustre abierto, y se refiere a la litología de las unidades como lodolita, caliza micrítica lodosa, ópalo y arena de grano fino. Los términos empleados por Caravella (1985) son más acordes al grado de litificación de los sedimentos, que aquellos empleados en los mapas de INEGI, ya que con la excepción de las rocas silicificadas, las unidades tienden a ser deleznables.

\section{METODOLOGÍA}

La investigación se desarrolló en campo y laboratorio. Durante las campañas de trabajo de campo se hizo cartografía geológica encaminada principalmente a establecer la distribución y naturaleza de los sedimentos de relleno de cuenca y se obtuvieron datos estructurales y muestras representativas de las distintas litologías.

La descripción y clasificación de las litologías encontradas se hizo con base en observaciones efectuadas en campo y en los resultados obtenidos con la técnica del hidrómetro para cuantificar los sedimentos finos en muestras colectadas. Las pruebas con el hidrómetro implementadas en este trabajo se basan en la metodología de la Norma Oficial Mexicana NOM-021-RECNAT-2000 (NOM, 2000) y se aplicaron en material no litificado. Dicha norma señala que se puede utilizar material que incluya arena, ya que partículas con tamaño aparentemente de arena pueden estar constituidas en realidad por partículas más finas agregadas, por lo que la técnica emplea disgregantes químicos y físicos. Para este trabajo se utilizó el material que pasó la malla 40 (abertura $0.420 \mathrm{~mm}$ ), de acuerdo con la clasificación granulométrica de Wentworth (1922), y el material que se empleó para esta prueba incluye arena media y fina, además de limo y arcilla. A este material se le eliminó la materia orgánica con peróxido de hidrógeno como indica la norma y se procedió a licuar los sedimentos para separar las partículas finas agregadas (pseudo-arenas). Una vez establecida la granulometría y el porcentaje de las fracciones finas y gruesas en las muestras, se dio un nombre generalizado a la muestra como arena-limosa, sedimento limo-arcilloso, entre otros, siempre dejando al inicio del término descriptivo la fracción presente en mayor cantidad. A estos nombres siempre se le agregó "carbonatado" en aquellas muestras que mostraron efervescencia con $\mathrm{HCl}$ diluido y "silícea" si parte del estrato en que se tomó la muestra presentaba un aspecto de ópalo o calcedonia.

En muestras litificadas se hizo la clasificación petrográfica de acuerdo con los criterios de Folk (1969). Se utilizaron también smear slides (e.g., Schnurrenberger et al., 2003) para reconocer los bioclastos en muestras no litificadas. Para describir el tamaño de grano se empleó la escala de Wentworth (1922) y Doeglas (1968). La denominación del color de los depósitos se basó en Goddard et al. (1963). Para la terminología del espesor de capas y láminas se utilizó el esquema sugerido por Stow (2005). En el laboratorio también se elaboraron con QGis los mapas hidrológico e hipsográfico del área de estudio y de sus alrededores, empleando como base información hidrológica del sistema SIATL (INEGI, 2010; <http://antares.inegi.org.mx/analisis/ red_hidro/siatl/>) y la altimetría de los modelos digitales de elevación de la misma agencia.

Los análisis para obtener la edad U-Pb de los circones se realizaron en cinco muestras de ceniza de caída, una ignimbrita y en una arena. Los análisis se hicieron con un sistema de ablación láser Resolution M50 (LPX Pro) acoplado a un ICP-MS cuadrupolo Thermo iCap Qc en el Laboratorio de Estudios Isotópicos (LEI) del Centro de Geociencias, Universidad Nacional Autónoma de México (UNAM). Cada análisis de circón tuvo una duración de $60 \mathrm{~s}$, de los cuales, $15 \mathrm{~s}$ correspondieron a la adquisición del blanco, $30 \mathrm{~s}$ al material producto de la ablación y $15 \mathrm{~s}$ posteriores a la ablación (washout). El diámetro del haz utilizado en el caso de los circones fue de 23 micrómetros con una distribución 
de energía por área (fluencia) cercana a $6 \mathrm{~J} / \mathrm{cm}^{2}$ utilizando una tasa de repetición de $5 \mathrm{~Hz}$. Los detalles de la optimización del espectrómetro de masas están descritos en Solari et al. (2010) y Ortega-Obregón et al. (2014).

El empleo de las edades de los circones de cenizas acumuladas en lagos no está exento de complicaciones. En su trabajo, Boygle (1994, 1999) demostró que las edades de tefras depositados en lagos no siempre son registros geocronológicos confiables de la edad de la actividad volcánica, y por ende de la edad de la secuencia de los sedimentos que la contienen. Se ha observado que tefra acumulada en lagos pequeños (varios cientos de metros de diámetro) es un mejor indicador de la actividad volcánica, mientras que capas de ceniza provenientes de lagos extensos (un par de decenas de kilómetros o más de diámetro), alimentados por cuencas hidrográficas grandes, comúnmente están formadas por ceniza acarreada por los ríos y/o mezclada por corrientes adentro del lago (Bertrand et al., 2014). Ese acarreo, en el caso de las cenizas de la paleocuenca de Teocaltiche, causó que en muchas de ellas exista una contribución importante de circones detríticos provenientes de las rocas volcánicas de la SMOc. Otra fuente importante de circones en algunas muestras son fragmentos accidentales de la roca encajonante alrededor del conducto volcánico, que fueron incorporados en la nube eruptiva. Por ese motivo, siempre se emplearon los conjuntos de circones más jóvenes en cada muestra para inferir la edad de depósito. Cabe señalar que en las siete edades reportadas en este trabajo solo se incluyeron tobas que no presentaron complicaciones en la interpretación de las edades. En todas las edades U-Pb reportadas se emplearon los resultados obtenidos en los circones más jóvenes y que cumplen con el requisito de tener una discordancia entre $-5 \mathrm{y}+30$ por ciento (resaltado en azul claro en el Anexo S1 del Suplemento Electrónico). Una excepción a lo anterior es la muestra 63B, que es de origen detrítico. En ella hay cuatro circones que, en comparación con otros analizados de la misma muestra, son más jóvenes, y aunque solo uno cumple con los parámetros de discordancia, también se tomaron en cuenta los otros tres, para poder acotar mejor la edad máxima de depósito.

En la Figura 4 se muestra un esquema conceptual, idealizado, que incluye lo que se cree fueron los principales elementos involucrados en la acumulación de los sedimentos adentro de la cuenca, asícomo la ubicación y naturaleza de las posibles fuentes del material detrítico en ella. En el diagrama se representan de manera esquemática las tres provincias geológicas que confluyen en el área, los elementos estructurales inferidos que originaron la paleocuenca y una caricatura de las redes de drenaje que aportaron los sedimentos, distinguiendo entre estas al río axial y a corrientes transversales en los bloques de techo y piso de un semigraben. La imagen ilustra también un esquema idealizado de los principales ambientes de depósito, dividiéndolos en ambientes subaéreos, transicionales y lacustres. El diagramaes también una clave de los términos empleados en este trabajo.

\section{GEOMORFOLOGÍA E HIDROGRAFÍA DE LA CUENCA}

El análisis de la geomorfología e hidrografía del área de estudio y sus alrededores, junto con el de los mapas de geología regional (e.g., Sánchez-González et al., 1998), permitieron inferir la posible extensión

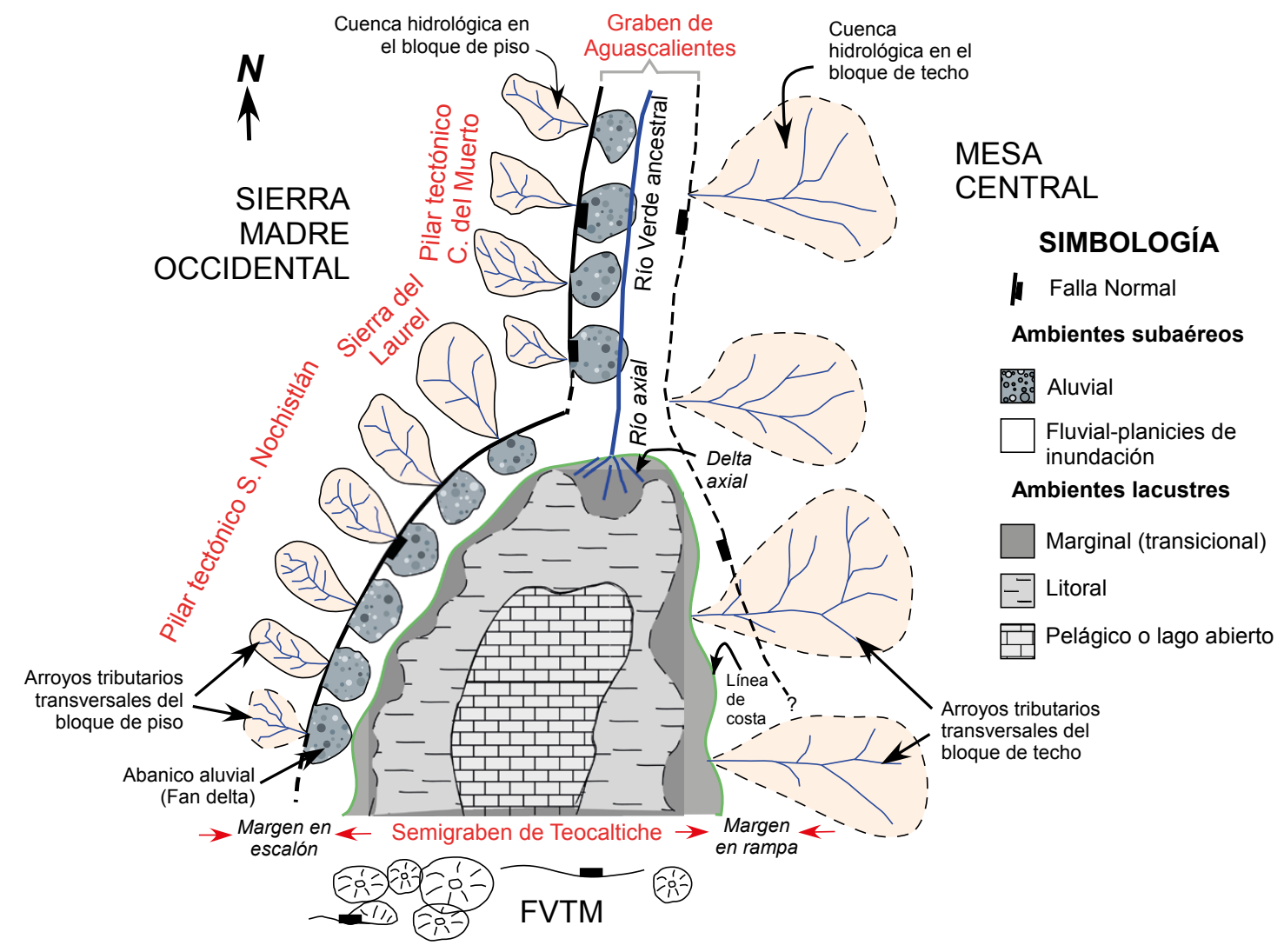

Figura 4. Esquema conceptual de la paleocuenca de Teocaltiche. Se muestra de manera simplificada los principales elementos involucrados en la formación de la cuenca y las posibles fuentes de sedimentos, los cuales provenieron de los bloques de techo y piso del semigraben, y fueron transportados por un río axial (paleo río Verde) y por ríos transversales. También se bosquejan los ambientes sedimentarios de primer orden presentes en el paleolago. FVTM = Faja Volcánica Transmexicana. Adaptado y simplificado de Leeder y Jackson (1993); Platt y Wright (1991) y Bank et al. (1995). 
y la influencia del control estructural en los límites de la paleocuenca. En las Figuras 2 y 5 se observa que el área de estudio es en parte una región que en el pasado fue aproximadamente plana y suavemente inclinada y que la erosión fluvial ha labrado una red fluvial con un arreglo dendrítico a partir del cauce principal del actual río Verde. La parte norte del graben de Aguascalientes en la Figura 5 se caracteriza por tener una densidad de drenaje relativamente baja mientras que en el área entre Teocaltiche, Yahualica y San Juan de los Lagos la densidad es mayor y la topografía está formada por lomeríos suaves, muchas veces con cimas planas. El arreglo dendrítico de la red refleja una resistencia baja a la erosión de los sedimentos fluvio-lacustres sin litificar o pobremente consolidados. Hacia el sur, en el área de El Zapotillo, al oriente de Yahualica, la corriente del río Verde se atrinchera (entrenched river), excava en el lecho rocoso y este río axial deja de tener planicies de inundación, que son notablemente mejor desarrolladas entre Belén del Refugio y San Nicolás (Figuras 5 y 6). Los afluentes transversales del río Verde son ligeramente más cortos hacia el poniente (Figura 2), mientras que los del oriente son más largos, lo que indica una asimetría en la cuenca consistente con el control que ejerce el semigraben en la red fluvial. Los tributarios al occidente del río Verde son, en su parte alta, ríos que drenan el bloque de piso del semigraben de Teocaltiche y del graben de Aguascalientes, mientras los tributarios del lado sudoriental fluyen sobre el bloque de techo del semigraben (Figura 2 y 4). La extensión total del área de acumulación en la cuenca

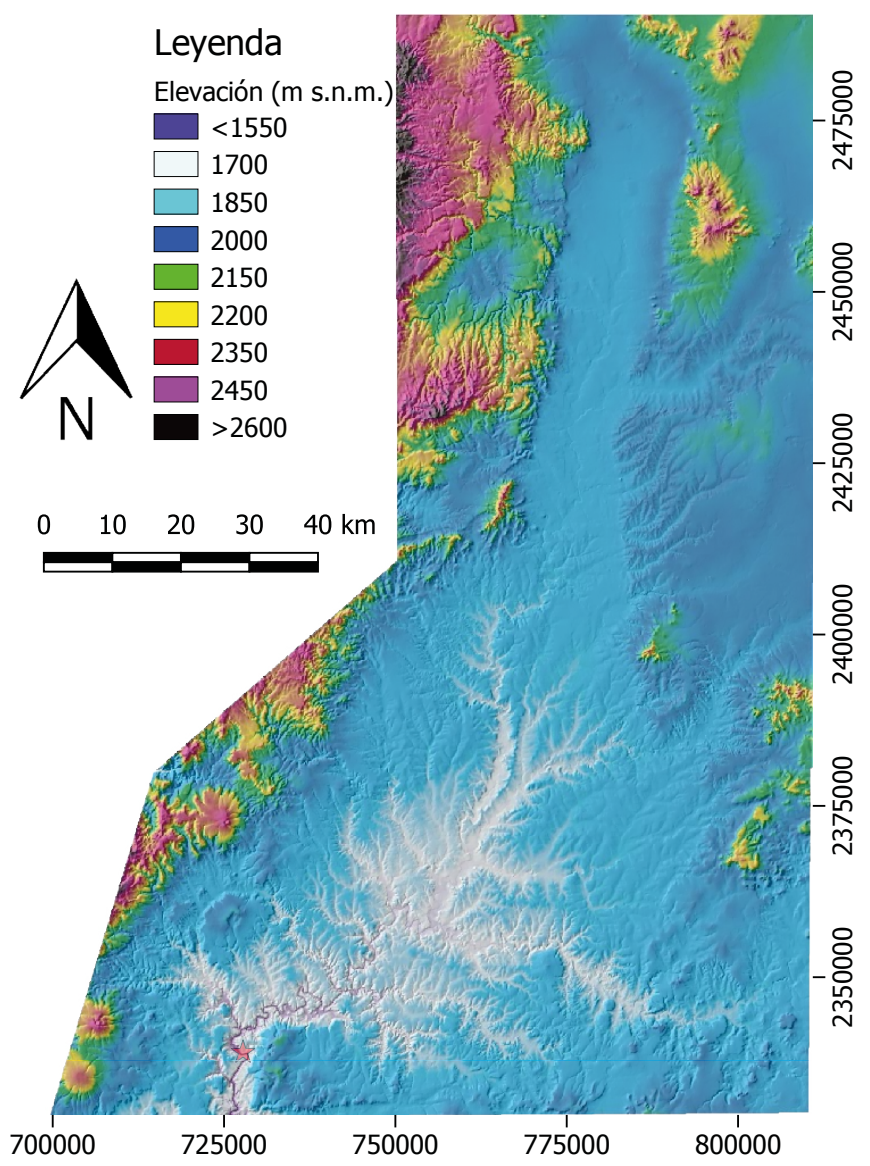

Figura 5. Mapa hipsográfico en el que se observa que la red hidrográfica es más densa y la profundidad de los cauces es mayor hacia el sur del área, cerca de El Zapotillo, mientras que el relleno de graben al norte de la ciudad de Aguascalientes está considerablemente menos erosionado. El mapa se localiza en el cuadrante 13Q con un DATUM WGS 84. fue delimitada aproximadamente mediante trabajo de reconocimiento geológico a lo largo de las carreteras y caminos (Figura 6). El áreafuente de donde procedieron en el pasado los sedimentos clásticos no puede establecerse con precisión, pero a manera de ensayo se simuló bloquear el cauce del río Verde actual mediante el simulador SIATL (INEGI, 2010) en el sitio en donde se encuentra la presa El Zapotillo, obteniéndose así la porción de la cuenca hidrográfica (Figuras la y 2) que aportaría el agua y clastos a un lago así formado.

El control estructural en el lado occidental de la paleocuenca lo ejercen los pilares tectónicos de las sierras de Nochistlán, El Muerto o Picacho y del Laurel (Figuras 4 y 5). Hacia el oeste del área de estudio, el relieve es más abrupto, con altitudes que van desde aproximadamente 1550 metros sobre el nivel del mar (m s.n.m.) en la parte baja del valle hasta $2580 \mathrm{~m}$ s.n.m. en la cima de las montañas. Las porciones más altas de esas sierras, así como los frentes de montaña, tienen direcciones preferenciales NNE en el sector norponiente del área y cambian a NESW en el surponiente, posiblemente reflejando un control estructural (e.g., Aranda-Gómez, 1989). En el sector oriental del área de estudio el relieve es más bajo porque la diferencia entre las altitudes de los valles y las sierras es menor y no existen cadenas de montañas continuas, sino que el terreno elevado se presenta como promontorios aislados o sierras pequeñas (¿vestigios erosivos?) con elevaciones máximas hasta de 2350 m s.n.m. (Figura 5). En su conjunto esas partes elevadas tienen una dirección similar a la sierra de Nochistlán al poniente (Figura 5) y en los mapas geológicos regionales se les muestra bordeadas por fallas normales inferidas (Sánchez-González et al., 1998).

Cabe hacer notar que la sinuosidad del frente de montaña en la parte oriental de la Sierra de Nochistlán es mayor que aquella en las sierras El Laurel y El Muerto (Figura 5), lo que de acuerdo a los criterios discutidos por Burbank y Anderson (2001) sugiere que el semigraben de Teocaltiche ha permanecido inactivo por un mayor tiempo que la falla maestra al occidente del graben de Aguascalientes, o bien que parte de ese límite tectónico está enmascarado por depósitos volcánicos más jóvenes. Esto último es especialmente evidente en los alrededores de Yahualica, en donde existen escudos volcánicos continentales del Mioceno (Figura 5).

\section{EL RELLENO SEDIMENTARIO DE LA PALEOCUENCA}

La unidad aquí llamada secuencia sedimentaria-volcánica de la cuenca de Teocaltiche es el relleno parcial de la paleocuenca Teocaltiche, la cual se encontraba en las depresiones tectónicas asociadas al semigraben de Teocaltiche y al graben de Aguascalientes (Figuras 4 y 5). La geomorfología actual sugiere que estas dos estructuras se unen y, a juzgar por la sinuosidad de los frentes de montaña (Keller y Pinter, 1996) se cree que sus fallas maestras están inactivas desde hace tiempo, probablemente desde el Mioceno tardío (Figura 5). Por otro lado, el sistema de depósito parece haber sido continuo en ambas fosas durante al menos la fase final de la sedimentación, ya que actualmente no existe una barrera topográfica que sugiera una separación o barrera física entre las dos estructuras.

En términos generales, la secuencia sedimentaria-volcánica tiene una gran variabilidad litológica tanto horizontal como vertical. Los cambios laterales están claramente asociados a variaciones en los ambientes de depósito, que deben presentarse de manera natural en distintas partes de la cuenca (Figuras 4 y 6 ) y las variaciones verticales documentadas en muchas localidades (ver columnas estratigráficas en el Anexo S2 del Suplemento Electrónico) se atribuyen a actividad tectónica en las fallas normales que delimitaban a la depresión topográfica y/o a cambios climáticos a largo plazo acaecidos durante la historia de la cuenca, que permitieron cambios en el espacio de "acomodación" para 


\section{LEYENDA}

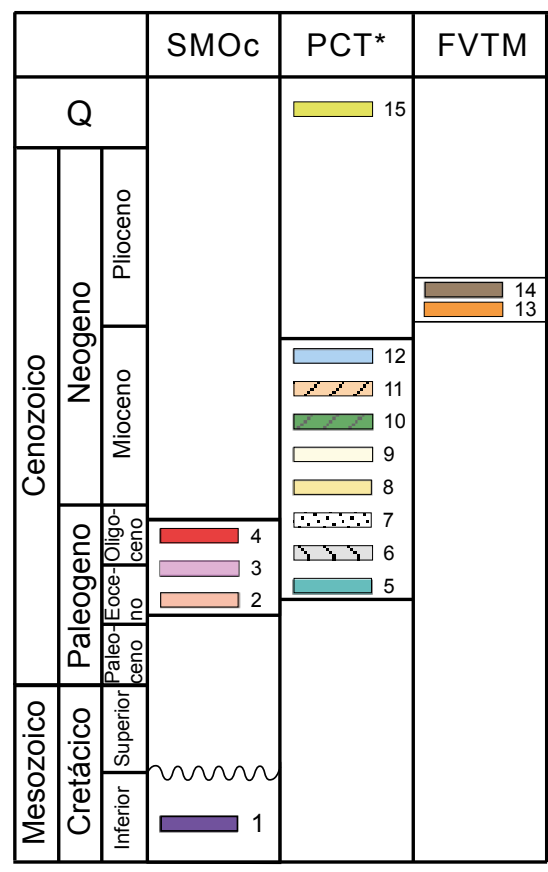

Símbolos

7 Rumbo y echado

$\nwarrow$ Dirección paleocorriente

6 Falla normal

6 Falla normal inferida

mDisconformidad

- Edades U/Pb en Ma

- Pozo "EI Potrero - El Zacate"

Fósil molar Calippus hondurensis

$\square$ Localidades citadas en el texto

* Paleocuenca Teocaltiche o

secuencia sedimentaria - volcánica

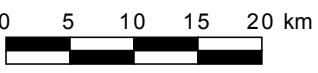

2330000

716000

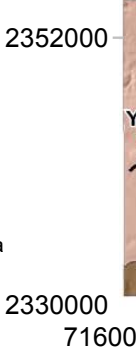

A

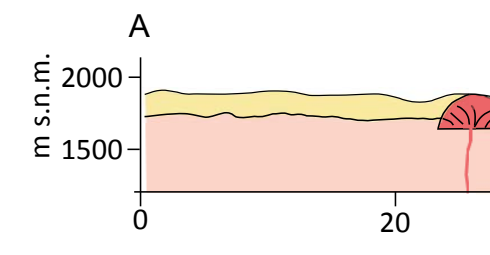

$20 \quad 40$
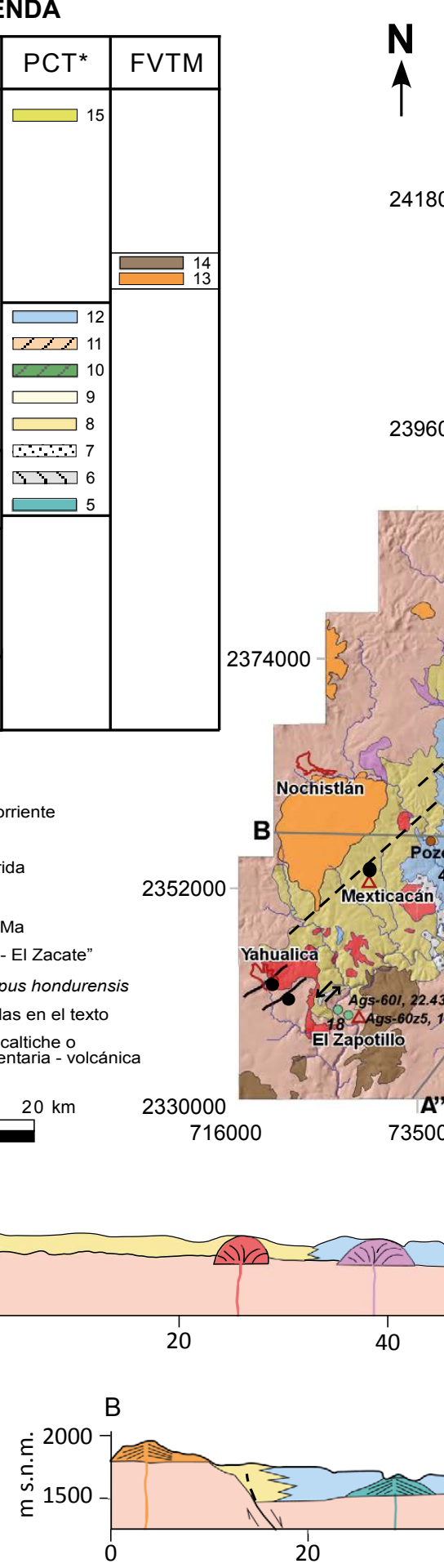

2374000

A

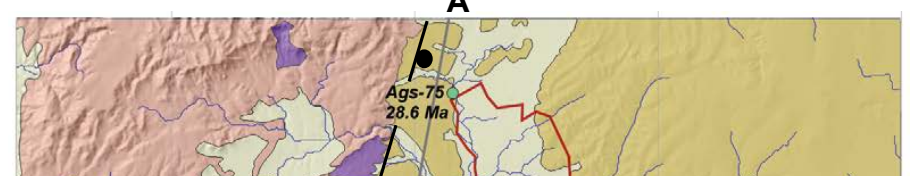

2418000

2396000

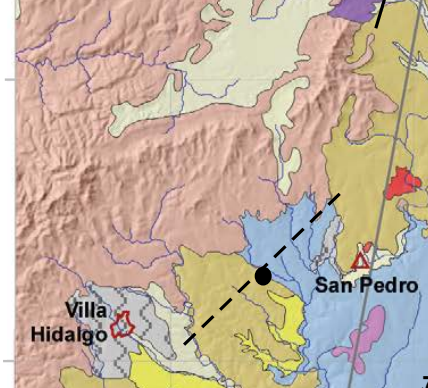

Aguascalie

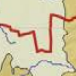

Aguascalientes

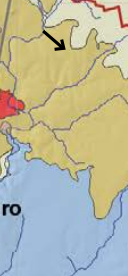

\Che
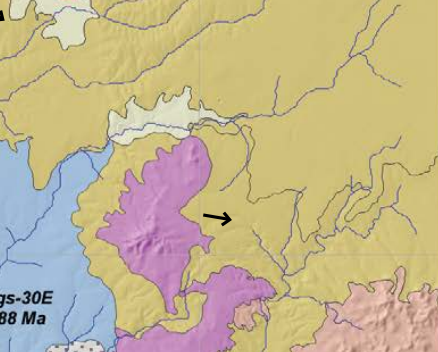

Encarnacións ${ }_{\text {de Diaz }}$

ㄴ.
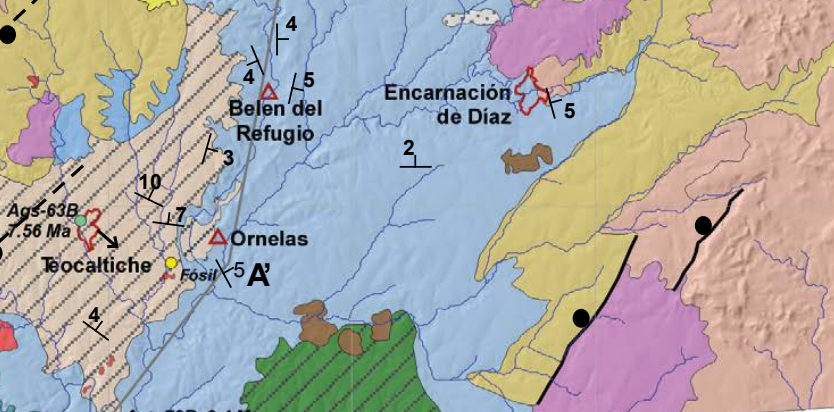
los sedimentos, de manera semejante a lo documentado por Lahiere (1982) y López (1991) en la cuenca de Juchipila.

En la secuencia sedimentaria, dependiendo de la ubicación geográfica adentro de la cuenca (Figura 6), se pueden distinguir sedimentos dominantemente: 1) clásticos, 2) precipitados químicos o bioquímicos y 3) sedimentos mixtos (una combinación de clásticos y precipitados). Los sedimentos clásticos son depósitos que varían de grano grueso (gravas) a fino (arena, arena-limosa, limo, limo-arcilloso), son epiclástico-volcánicos, ya que casi la totalidad de sus componentes es derivada de productos de la SMOc y oscilan de débilmente de litificados a sin litificar. Se presentan en depósitos con capas irregulares, con estratificación cruzada o planar, en algunos lugares con canales internos. Algunos de los depósitos de grava muestran imbricación de clastos que permiten inferir la dirección de flujo local (Figura 6). Las rocas dominantes en los clastos son fragmentos de ignimbritas y lavas félsicas (riolíticas a riodacíticas) y, en menor proporción, fragmentos de lavas intermedias. Algunos de los clastos de andesita son interpretados como derivados de lavas en la sucesión de la SMOc y otros, especialmente en la parte meridional de la cuenca se cree que provienen de rocas de la sucesión temprana de la FVTM. Los depósitos clásticos se encuentran esencialmente en la periferia del depocentro y los de grano grueso se concentran en mayor medida hacia el norte y occidente del área (Figuras 4 y 6), en las márgenes de la depresión tectónica y en los sitios en donde descargaba el río axial y los afluentes transversales del bloque de piso adentro del lago (Figuras 4 y 6). Todos estos componentes se considera que son principalmente derivados de la erosión de los productos de la provincia volcánica de la SMOc que cubrían la mayor parte (¿o posiblemente toda?) el área fuente de sedimentos para la cuenca de Teocaltiche. Cabe señalar que la porción meridional de la MC actual estuvo sepultada por productos volcánicos de la SMOc (e.g., Ortega-Gutiérrez et al., 1992). Los sedimentos de grano medio a fino, se encuentran al sur del área de estudio, y son depósitos de arena masiva, sin litificar o parcialmente litificada, comúnmente cruzados por enjambres de diques clásticos delgados, compuestos por arena fina y/o limo. En general, los sedimentos clásticos son interpretados como depósitos de ambientes fluviales o aluviales o deltas litorales, ya sea de la corriente axial o de tributarios transversales (Figuras 4 y 6).

Los depósitos de precipitados químicos o bioquímicos son sedimentos calcáreos de agua dulce, de color blanco a amarillo pardo, con diferentes grados de alteración diagenética, que causan que localmente los sedimentos varíen de bien litificados a parcialmente litificados. En muchos sitios la presencia de concreciones de sílice y reemplazamiento selectivo es común, y en otros se presentan icnofósiles (¿Domichnia?), que tienen formas cilíndricas rectas y son perpendiculares a la estratificación: (Gámez-Vintaned y Liña, 1996). Ostrácodos y/o algunas diatomeas también son comunes. Los precipitados químicos y bioquímicos se presentan en capas masivas de 1.5 a $2 \mathrm{~m}$ de espesor. En otros lugares muestran una estratificación paralela y difusa. Estos sedimentos se observan principalmente en el centro y sur del área de estudio, en donde dominaban los ambientes pelágico y de lago somero (Figuras 4 y 6). En algunos sitios en la secuencia también hay horizontes de paleosuelo como capas con forma irregular, delgadas a medianas, de color rojizo y amarillo claro, en ocasiones bioturbadas y/o con fragmentos de raíces. Varían de débilmente litificadas a sin litificar y muestran contactos discordantes principalmente hacia su cima. Estos paleosuelos se observan intercalados en la secuencia sedimentaria-volcánica principalmente en la zona meridional de la paleocuenca (Figura 6) y se les considera representativos del ambiente transicional, cercano al litoral y sublitoral del lago, cuyo lecho estuvo periódicamente expuesto por fluctuaciones en el nivel del agua.

Los sedimentos mixtos son margas formadas por arena fina, limo, arcilla y carbonato de calcio. Tanto las margas como los precipitados varían de débilmente litificados a sin litificar y en ocasiones están bien litificados ya que en muchos sitios se observan parcial a totalmente silicificados (reemplazados por ópalo: Caravella, 1985). Las capas tienen espesores de 0.3 a $1.50 \mathrm{~m}$ y en ocasiones están bioturbadas, con rizolitos. En otros sitios se observan microbialitas (¿biomicritas?). Estos sedimentos mixtos tienen como característica principal que en el extremo poniente del área de estudio son de color verde claro, mientras que en la parte oriental tienen paleosuelos intercalados hacia su cima (Figura 6). En general, los sedimentos mixtos son interpretados como acumulados en ambientes lacustre marginal o en deltas litorales (Figura 4 y 6).

Los depósitos volcánicos intercalados en diferentes niveles estratigráficos en la secuencia sedimentaria son dominantemente capas de ceniza de caída, félsica a intermedia, sin litificar o parcialmente litificadas, que se caracterizan por ser masivas o débilmente laminadas. El espesor de las capas varía de $1 \mathrm{~cm}$ a $13 \mathrm{~cm}$ y su granulometría es de ceniza fina a media. Las capas rara vez están formadas por lapilli, lo que sugiere una distancia significativa a su fuente. Generalmente las tobas están compuestas por esquirlas de vidrio y las más gruesas por fragmentos pequeños de pómez. En al menos una localidad, cercana a Belén del Refugio (13Q 13767598E, 2383903N) se observó una capa de ceniza con rasgos que se interpretan como bioturbación. Las capas de ceniza se encuentran distribuidas en casi toda la paleocuenca, pero son más escasas hacia el norte donde hasta ahora solo se identificaron en una localidad al norte de la ciudad de Aguascalientes. En general, al menos en las porciones más jóvenes de la secuencia sedimentaria, se les interpreta como formadas por material piroclástico extracuenca. En unos cuantos sitios se observaron derrames de lava, de composición intermedia a máfica, intercalados con los depósitos sedimentarios, por lo que se cree que hubo actividad volcánica intracuenca en volcanes que posiblemente son outliers de la FVTM (Figura 6: litología 5). En la zona sur del área, en las paredes del cañón del río Verde, cerca de El Zapotillo, en la cima de la sucesión sedimentaria-volcánica expuesta se documentó una ignimbrita parcialmente soldada (muestra Ags-60 Z) y una capa de ceniza rosa intercalada cerca de la base de la sucesión (muestra Ags-60 I) (Figura 6 y Anexo S2). El significado de estas rocas ígneas con relación a la evolución de la cuenca, será discutido más adelante.

El espesor total de la secuencia sedimentaria-volcánica es desconocido. En la localidad de El Zapotillo (13Q 726541E, 2340466N), en la porción meridional del área de estudio, alcanza solo $70 \mathrm{~m}$ (Anexo S2). En la parte norte, donde los ríos actuales aún no han erosionado mucho la secuencia (Figura 5), hay afloramientos con $5 \mathrm{~m}$ de espesor expuesto. Cerca de Mexticacán (Figura 6), con base a los espesores reportados en un pozo de agua llamado Potrero-El Zacate (Figura 6, coordenadas UTM del brocal del pozo 13Q 734973E, 2357032N) que fue perforado por el IMTA (2007), la secuencia sedimentaria-volcánica localmente tiene un espesor de $90 \mathrm{~m}$, por lo que sumando la diferencia de altitud entre el brocal del pozo y la cima de las colinas circundantes, en donde afloran los sedimentos, se estima que en ese lugar el espesor mínimo del relleno sedimentario es de $160 \mathrm{~m}$. Sin embargo, se ignora si el lecho rocoso encontrado en la perforación es la base de la secuencia sedimentaria-volcánica o si se trata de un cuerpo de roca volcánica intercalado en ella.

\section{EDAD DE LOS SEDIMENTOS}

\section{Biocronología}

La edad biocronológica de un afloramiento de la sucesión sedimentaria acumulada en un ambiente marginal se infirió a partir de un maxilar de Calippus hondurensis, que corresponde a lo más temprano 
del Henfiliano tardío (Hh2 6.95 - 5.6 Ma; Carranza et al., 2013 La localidad en donde se colectó el fósil (Figura 6) es un banco de material con una profundidad máxima de $\sim 10 \mathrm{~m}$, ubicado en las coordenadas 13Q 759219E - 2369080N).

\section{Paleontología Sistemática}

Orden Perissodactyla Owen, 1848

Family Equidae Gray, 1821

Genus Calippus Matthew and Stirton, 1930

Calippus (Grammohippus) hondurensis (Olson and McGrew, 1941)

Material referido. Fragmento de paladar (Figura 7); el lado izquierdo, conserva la parte lingual y protocono de P4, los molares M1, M2 y M3 no presentan metastilo, mesostilo y parastilo, el resto de la superficie oclusal está completo. En el lado derecho se conserva la superficie oclusal del M2, pero al igual que los molares del lado izquierdo faltan el metastilo, parastilo y mesostilo. P2 aislado, completo.

Descripción. Los molares tienen la curvatura pronunciada y su grado de hipsodoncia es característico de Calippus, con la altura de la corona baja. El protocono es alargado, con forma tabular, paralelo al borde lingual del maxilar. El borde lingual es plano y paralelo al borde del maxilar. El borde anterior no presenta talón, solo una débil constricción. El borde posterior es lobular en todos lo molares.

Las fosetas son simples, los pliegues del borde anterior y posterior de la prefoseta y postfoseta han desaparecido con el desgaste; lo mismo ha ocurrido con el hipocono que no se observa y tampoco existe vestigio del surco hipoconal que está cerrado completamente, lo que es normal en molares de individuos adultos viejos.

El P2, que se encuentra dentro de los especímenes colectados,

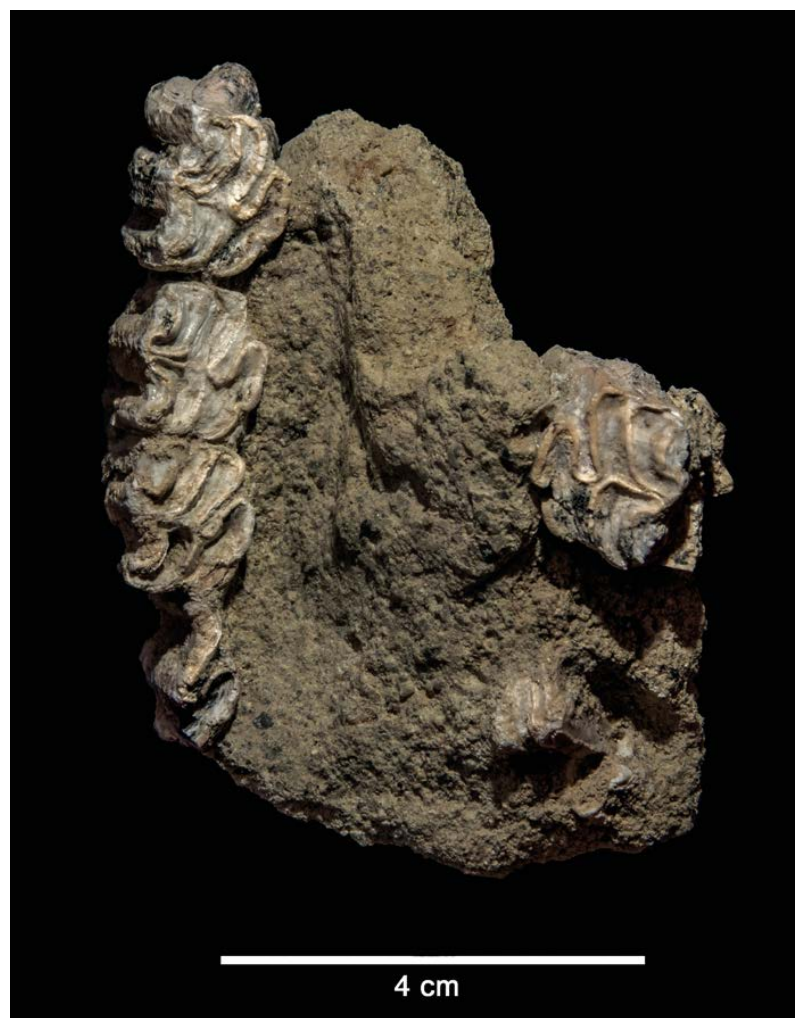

Figura 7. Fragmento de mandíbula de Calippus hondurensis. Véase descripción en el texto. se caracteriza por tener un anterostilo corto, el protocono alargado, las fosetas sin pliegues, el hipocono ha desaparecido sin vestigios del surco hipoconal.

Discusión y comparación. El paladar es asignado al género Calippus hondurensis por las características del protocono que es aplanado y su borde lingual paralelo al margen lingual del maxilar, a diferencia de otros géneros que tienen el protocono lobular y su borde posterior inclinado dirigido al borde lingual del maxilar. En la comparación con otros registros de México, el ejemplar de Teocaltiche, es similar a los molares descritos del paladar MPGJ 1704 referido a Calippus (Grammohippus) hondurensis y otros especímenes ilustrados en el trabajo de Carranza-Castañeda et al. (2013), los cuales corresponden a diferentes estados ontogenéticos y se observa la variabilidad que presentan y confirma la asignación de este material de Teocaltiche.

Distribución en México. Calippus (Grammohippus) hondurensis se ha descrito de la fauna de Sietla en el Estado de Hidalgo, en donde fue asignado al Henfiliano temprano; la fauna de La Presa en San Miguel de Allende que se ha referido al Henfiliano temprano más tardío y la mayor representación de este género se ha colectado y descrito de la fauna de Juchipila asignada a la edad de Henfiliano temprano (Carranza-Castañeda et al. 2013).

\section{Geocronología U-Pb}

Todas las edades isotópicas obtenidas en este estudio se obtuvieron por el método $\mathrm{U}-\mathrm{Pb}$ en circones separados de muestras de ceniza de caída intercaladas en los sedimentos fluvio-lacustres, de una arena y de una ignimbrita félsica soldada que cubre a parte de la secuencia sedimentaria-volcánica en la parte meridional del área (Tabla 2). Los datos obtenidos de las tobas de caída y de la ignimbrita son tratados como edades de cristalización y se consideran como "edades instantáneas" dentro del proceso de relleno de la cuenca. Los datos analíticos completos se reportan en el Anexo S1 del Suplemento Electrónico.

Las edades isotópicas obtenidas indican que la sedimentación en las depresiones tectónicas del graben de Aguascalientes y el semigraben de Teocaltiche sucedió en el lapso comprendido al menos entre $\sim 28$ y $\sim 7 \mathrm{Ma}$. La edad más antigua 28.6 $\pm 0.52 \mathrm{Ma}$ corresponde a la muestra Ags-75 (ceniza blanca fina con cuarzo y feldespatos), colectada a corta distancia al norte de la ciudad de Aguascalientes, en el poblado de Jesús María (Figura 6, Tabla 2) en una secuencia de sedimentos de origen fluvial que está claramente afectada por fallas cercanas al borde occidental del graben. La edad es una media ponderada, para la que se utilizó una población de seis circones, los más concordantes y jóvenes, obteniendo un MSWD de 1.18 (Figura 8a, Anexo S1). En la misma muestra se fecharon varios circones concordantes de $\sim 45 \mathrm{Ma}$ que son interpretados como xenocristales, posiblemente provenientes de rocas atravesadas por el conducto volcánico, que posiblemente estaba desarrollado, al menos en parte, en rocas del Complejo Volcánico Inferior de la SMOc.

La muestra Ags-60 I (ceniza rosa), con una edad de $22.43 \pm 0.94 \mathrm{Ma}$, proviene del extremo meridional del área y fue colectada cerca de la base de la secuencia fluvio-lacustre, que en ese lugar tiene un espesor mínimo cercano a $70 \mathrm{~m}$ y que está expuesta en el cañón del río Verde en el área de El Zapotillo (Figura 6, Tabla 2 y Anexo S2). La edad es el promedio ponderado obtenido de una población de los cuatro circones más jóvenes y concordantes del conjunto de granos analizados, con un MSWD de 2.3 (Figura 8b, Anexo S1). En la cima de la misma sección estratigráfica se colectó la muestra Ags-60 Z que es una ignimbrita soldada que sepulta parcialmente a la sucesión sedimentaria-volcánica en esa área (Anexo S2). Los seis circones más concordantes y jóvenes de Ags-60 Z, son consistentes con una edad de cristalización de $18.57 \pm 0.39 \mathrm{Ma}$, con un MSWD de 1.5 (Figura 8d, Anexo S1).

En la sección estratigráfica expuesta en las cercanías del poblado 
de San Nicolás, en las coordenadas UTM 13Q 753634E - 2356184N (Figura 6, Tabla 1 y Anexo S2), se colectaron dos muestras de ceniza intercaladas con litologías consistentes con la parte pelágica del lago (arcilla, marga y lodo micrítico). En la parte media de la columna expuesta se colectó la muestra Ags-72 J y unos $8 \mathrm{~m}$ estratigráficamente más arriba la muestra Ags-72 R. Las edades obtenidas del promedio ponderado, empleando los circones más jóvenes y concordantes, son de $19.44+0.56 \mathrm{Ma}$ y $9.47+0.21 \mathrm{Ma}$, respectivamente (Figura $8 \mathrm{c}$ y $8 \mathrm{e}$, respectivamente y Anexo S1).

Las muestras más jóvenes provienen de la parte media y norte del área con sedimentos lacustres. En la localidad del El Retoño (Figura 6, Tabla 2) se obtuvo una edad de 7.88 $\pm 0.19 \mathrm{Ma}$ en circones separados de una capa de ceniza (Ags-30 E) (Figura 8f, Anexo S1), mientras que la arena Ags-63 B dio una edad máxima, menos precisa, de $7.56 \pm 0.5 \mathrm{Ma}$ a partir de un grupo de cuatro circones detríticos (Figura 8g, Anexo $\mathrm{S} 1)$. Aunque solo se tiene un circón que cumple con el criterio de $<30 \%$ de discordia, existe otro (30.5\% de discordancia) que está cerca de la línea de discordia. Para precisar lo que pudiera ser la edad máxima de depósito, también se tomó como válido utilizar los circones que tienen discordancias ligeramente mayores a $30 \%$, pero que están muy próximos a la edad sugerida por el único circón que satisface el criterio $<30 \%$ de discordia. Suponemos que ese subconjunto de circones son, muy probablemente, comagmáticos entre sí. Bajo este supuesto se construyó una línea de discordia para obtener una edad de intersección inferior, que es lo que se presenta en la Figura $8 \mathrm{~g}$. Adicionalmente se hace notar que la edad de la muestra Ags-63 B es similar a la de la muestra Ags-30 E y cercana a la edad bioestratigráfica inferida para el fósil estudiado que se encuentra dentro del rango entre 6.8 y $4.75 \mathrm{Ma}$. Por los motivos señalados consideramos la edad de depósito en cuestión como razonablemente confiable. En esa misma muestra de arena se obtuvieron picos a 22, 28, 30, 47 y 52 Ma en el diagrama de probabilidad relativa. Las edades de las poblaciones más antiguas en la muestra son consistentes con los pulsos magmáticos registrados en la SMOc y en la MC (Nieto-Samaniego et al. 1996, Tristán-González et al. 2009).

El registro estratigráfico en el relleno de cuenca abarca un tiempo prolongado ( 21.04 Ma), que arranca después de la etapa inicial de formación del graben de Aguascalientes y se prolonga al menos hasta casi el límite Mioceno-Plioceno. Aunque las tobas son volumétricamente menos importantes que los sedimentos clásticos, precipitados

Tabla 2. Edades U-Pb (circón) de muestras colectadas en la paleocuenca Teocaltiche. Los análisis de U-Pb de cada circón se muestran en el Anexo S1.

\begin{tabular}{|c|c|c|c|}
\hline Muestra & Descripción & $\begin{array}{l}\text { Coordenadas } \\
\text { UTM 13Q }\end{array}$ & $\begin{array}{c}\text { Edad } \\
\text { (U-Pb en circones) }\end{array}$ \\
\hline Ags-63 B & $\begin{array}{l}\text { Arena verde - negra fina con } \\
\text { minerales magnéticos, cuarzo } \\
\text { y feldespato }\end{array}$ & $\begin{array}{l}750505 \mathrm{E} \\
2371222 \mathrm{~N}\end{array}$ & $7.56 \pm 0.6 \mathrm{Ma}$ \\
\hline Ags-30 E & $\begin{array}{l}\text { Ceniza gris fina con cristales } \\
\text { de cuarzo, feldespatos y } \\
\text { ferromagnesianos }\end{array}$ & $\begin{array}{l}773565 \mathrm{E} \\
2391916 \mathrm{~N}\end{array}$ & $7.88 \pm 0.2 \mathrm{Ma}$ \\
\hline Ags-72 R & $\begin{array}{l}\text { Ceniza gris fina con cuarzo y } \\
\text { feldespatos. }\end{array}$ & $\begin{array}{l}753634 \mathrm{E} \\
2356184 \mathrm{~N}\end{array}$ & $9.47 \pm 0.21 \mathrm{Ma}$ \\
\hline Ags-60 Z5 & $\begin{array}{l}\text { Ignimbrita bien soldada color } \\
\text { café, con fiammes negras y } \\
\text { cristales de cuarzo. }\end{array}$ & $\begin{array}{l}726541 \mathrm{E} \\
2340466 \mathrm{~N}\end{array}$ & $18.57 \pm 0.4 \mathrm{Ma}$ \\
\hline Ags-72 J & $\begin{array}{l}\text { Ceniza gris fina con cristales } \\
\text { de cuarzo y feldespatos }\end{array}$ & $\begin{array}{l}753634 \mathrm{E} \\
2356184 \mathrm{~N}\end{array}$ & $19.4 \pm 0.5 \mathrm{Ma}$ \\
\hline Ags-60 I & $\begin{array}{l}\text { Ceniza rosa fina con cuarzo, } \\
\text { feldespatos y minerales } \\
\text { magnéticos. }\end{array}$ & $\begin{array}{l}726541 \mathrm{E} \\
2340466 \mathrm{~N}\end{array}$ & $22.43 \pm 0.1 \mathrm{Ma}$ \\
\hline Ags-75 & $\begin{array}{l}\text { Ceniza blanca fina con cuarzo } \\
\text { y feldespatos. }\end{array}$ & $\begin{array}{l}774647 \mathrm{E} \\
2429999 \mathrm{~N}\end{array}$ & $28.6 \pm 0.5 \mathrm{Ma}$ \\
\hline
\end{tabular}

químicos y bioquímicos y sedimentos mixtos, son un registro de la actividad volcánica, tanto en el área ahora ocupada por la cuenca como en sus alrededores. Los datos obtenidos sugieren que esa actividad fue en pulsos separados por hiatos, lo que coincide a grandes rasgos con lo que se sabe acerca del desarrollo de las provincias volcánicas de la SMOc y la FVTM en la región. La edad de los pulsos volcánicos reconocidos en la secuencia de relleno de graben es de 1) 28 Ma, 2) 23-19 Ma, y 3 ) $~ 9-8 \mathrm{Ma}$ (Figura 9). Los dos primeros son correlacionables con pulsos en el Supergrupo Volcánico Superior de la SMOc (McDowell y Keizer, 1977) y el tercero al pulso magmático del Mioceno tardío al Plioceno temprano en la parte norte del FVTM (Ferrari et al., 2012).

\section{EVOLUCIÓN DE LA CUENCA}

En un análisis regional acerca de la evolución estructural de la porción centro-occidental de México, Nieto-Samaniego y colaboradores (1999) propusieron que un conjunto de fallas normales en dicha región iniciaron su actividad durante el Oligoceno y que tanto el foco del volcanismo como de la extensión se desplazaron desde la parte sudoriental de la MC hacia la costa del Pacífico a partir del Oligoceno. De acuerdo con este modelo, las fallas que bordean a la paleocuenca de Teocaltiche tienen una edad entre 29 y $27 \mathrm{Ma}$ (Nieto-Samaniego et al., 1999; Loza-Aguirre et al., 2008). Aquí se asume que una vez que comenzaron a operar las fallas normales inició la formación de depresiones que potencialmente pudieron actuar como cuencas sedimentarias de origen tectónico.

Los estudios sedimentológicos y estratigráficos de Lahiere (1982) y López (1991) en el graben de Juchipila demostraron que los sedimentos de relleno de graben están claramente afectados por fallas que cortan y desplazan a sedimentos con edades paleontológicas del Henfiliano y edades U-Pb de circones separados de capas de ceniza de caída intercalados con las capas fosilíferas demuestran que la sedimentación fluvio-lacustre estaba activa en el lapso comprendido entre aproximadamente 7.0 y $5.6 \mathrm{Ma}$ (Carranza-Castañeda et al., 2013). Aún más, cambios litológicos importantes en la secuencia de relleno de graben de Juchipila fueron interpretados como evidencia de actividad tectónica sin-sedimentaria (López, 1991). Cabe señalar que el alcance temporal del relleno de graben en Juchipila es poco conocido, porque todas las edades isotópicas publicadas han tenido como objetivo obtener la edad numérica de los fósiles estudiados por Carranza-Castañeda y colaboradores (2013). No se sabe si hay sedimentos de otras edades en la cuenca.

En los sedimentos de la paleocuenca de Teocaltiche las capas de ceniza de caída y/o retrabajada, contemporáneas a la sedimentación son muy comunes y se puede afirmar que en casi todos los afloramientos es posible identificar una o más capas de material volcánico. Esta circunstancia hace posible el fechamiento isotópico de los sedimentos y un análisis acerca de la posible evolución de la cuenca. Un resultado no previsto al iniciar esta investigación es el rango de edades del relleno de la cuenca, que prácticamente inició junto con la extensión en la región, lo cual es un hecho que no había sido reconocido en el área. Esto abre la posibilidad de comprender mejor la evolución de un sistema en donde sucedió agradación de sedimentos contemporánea con fallamiento y volcanismo en un ambiente continental del centro de México.

\section{POSIBLES CAUSAS DEL CIERRE DE LACUENCA}

Hasta ahora la única hipótesis acerca del evento que precipitó la formación del lago en la paleocuenca de Teocaltiche es aquella planteada por Caravella (1985), quien argumentó que el río Verde ancestral 

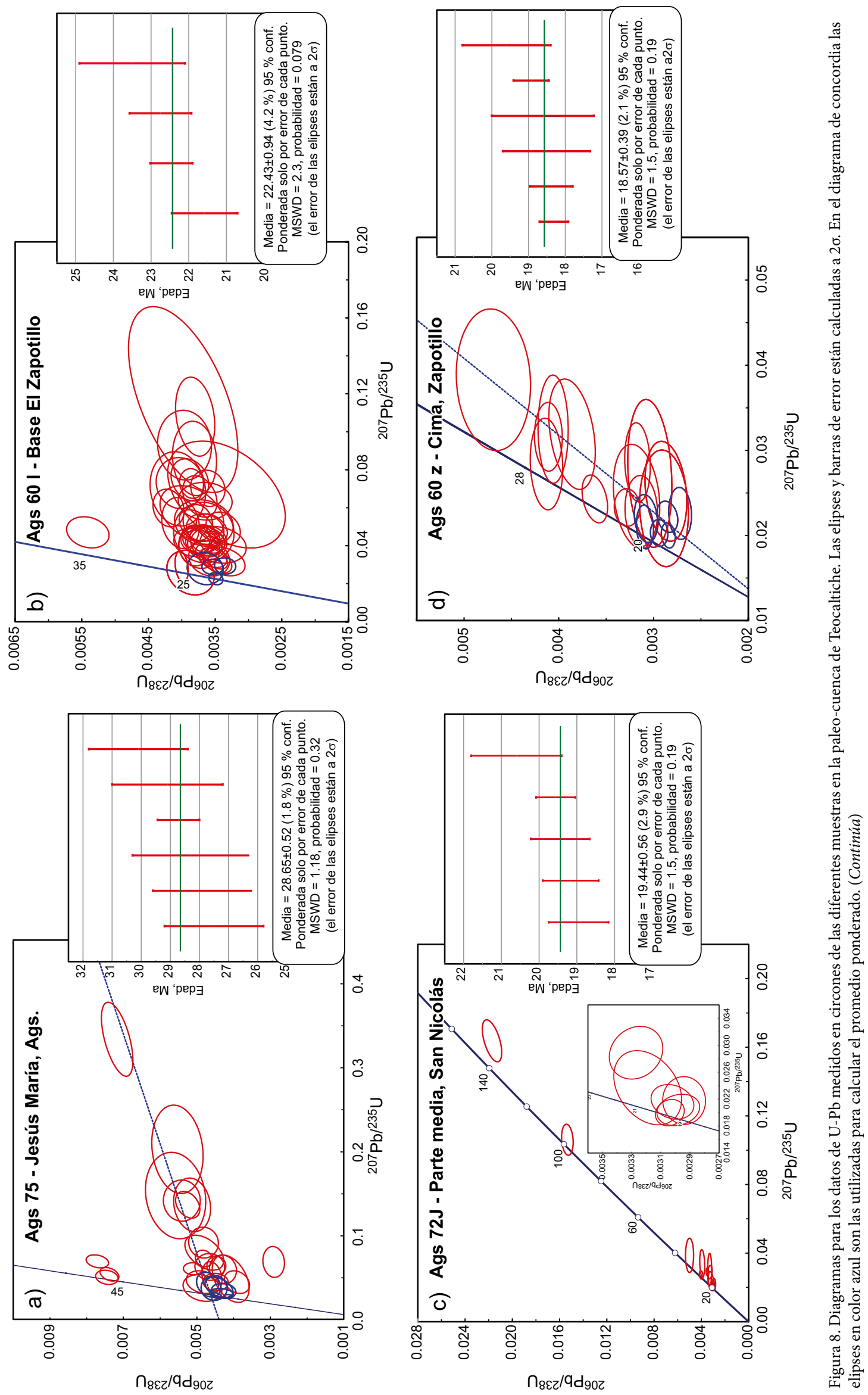


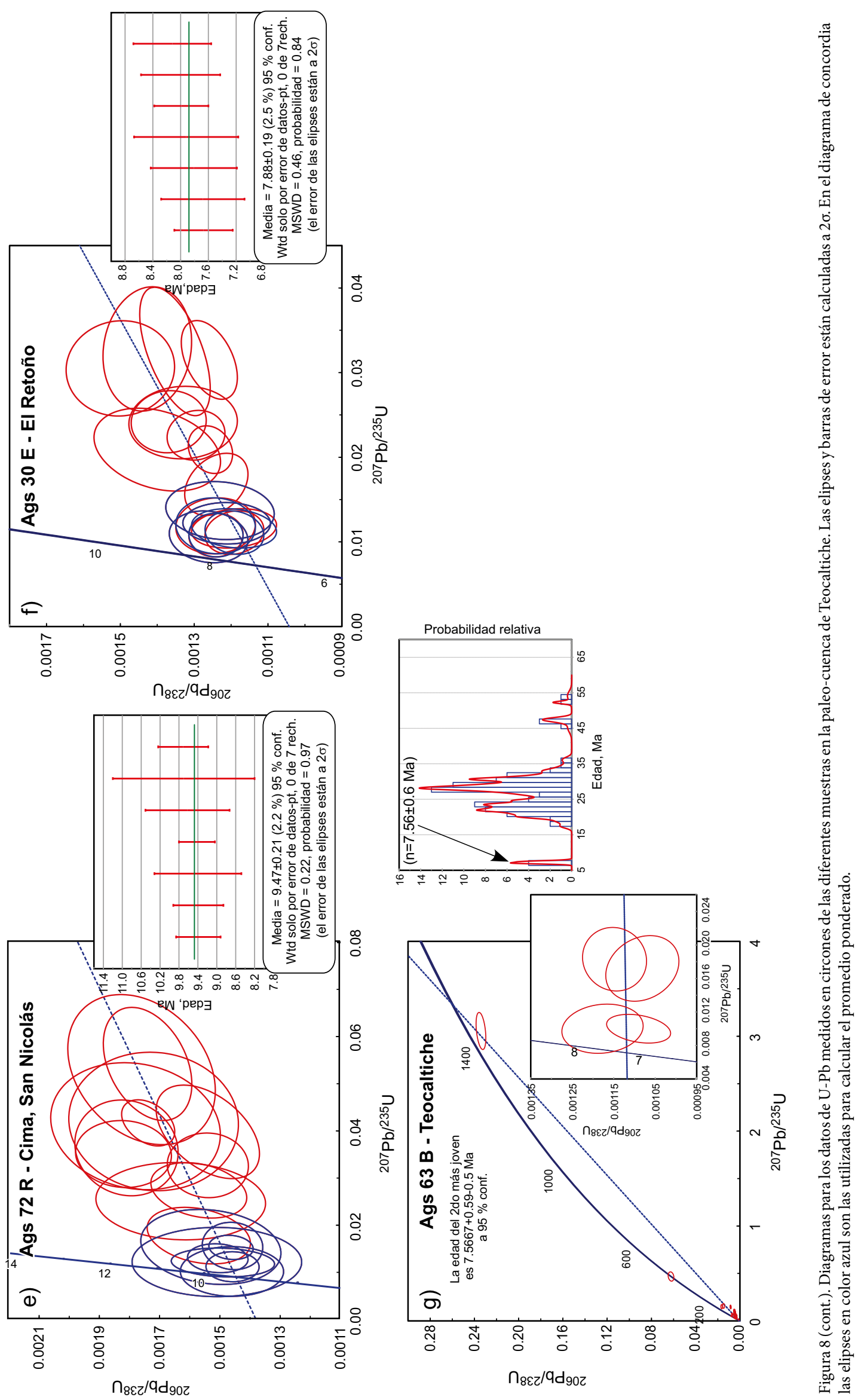


pudo ser represado "por depósitos volcánicos coalescentes" y desechó, de manera implícita, la posibilidad de que la causa haya sido relacionada con un evento tectónico (con base en la escasez de fallas en la parte de la cuenca que él investigó).

Factores como la distribución regional del relleno sedimentario, el alcance temporal de la secuencia sedimentaria, la presencia de volcanes $\mathrm{y}$ de sistemas de falla en las inmediaciones de la cuenca y el entorno tectónico regional de la misma pueden auxiliar en la resolución de esta cuestión, $\mathrm{o}$ al menos ayudar a plantear hipótesis alternativas para realizar investigaciones futuras.

La inspección visual de mapas de relieve sombreado, hipsográfico (Figuras 3 y 5) y de imágenes de satélite del área de estudio y sus alrededores, así como la consideración del rango de edades de los sedimentos y edades isotópicas de edificios volcánicos contiguos al borde meridional de la paleocuenca (Figura 3, localidades 5 y 6), llevan a concluir que los volcanes escudo y derrames de lava asociados a ellos cercanos a Yahualica (Figura 6) y el sistema de fallas normales E-W ubicado al sur de la Presa El Zapotillo (Figura 3) pudieron causar el bloqueo de la red de drenaje del río Verde ancestral, durante el Mioceno medio. Sin embargo, esta interpretación, casi obvia después de examinar la información mencionada, no es satisfactoria para explicar la parte de la secuencia de relleno de graben que se acumuló en el lapso comprendido entre las edades de la ignimbrita y toba de caída fechadas en la sección de El Zapotillo $(\sim 18$ y $\sim 22)$ y la edad más antigua ( 28 Ma) obtenida en la parte meridional del graben de Aguascalientes, porque esa parte de la sucesión antecede a las edades de los volcanes y sistemas de falla cercanos a Yahualica.

El hecho de que en otros grábenes de la parte meridional de la SMOc (Figura 10d) se hayan colectados mamíferos fósiles del Henfiliano (6.8 - 4.75 Ma) en sucesiones fluvio-lacustres (CarranzaCastañeda, 2006b y Carranza-Castañeda et al., 2013) indica que en al menos tres de esas cuencas tectónicas (Tlaltenango, Juchipila y Teocaltiche) existieron de manera sincrónica lagos perennes.

En el graben de Bolaños, ubicado al oeste de la fosa de Tlaltenango, también se ha propuesto la existencia de un paleolago durante el Neógeno (Scheubel et al., 1988). Una causa común para el bloqueo simultáneo aproximadamente a la misma latitud $\left(c a .21 .3^{\circ} \mathrm{N}\right)$ de al menos tres de las cuencas, requiere de un mecanismo de carácter regional. Al sur de la región en donde existieron los lagos se encuentran los rifts de Chapala-Tula y Tepic-Zacoalco (Johnson y Harrison, 1990), que son burdamente perpendiculares al sistema de fallas normales que afectan a la parte meridional de la SMOc. Ambas estructuras tienen evidencias claras de actividad tectónica reciente, pero no se sabe con precisión cuando comenzaron a operar. En el caso del rift Tepic-Zacoalco, para localidades en las cercanías de la presa La Yesca se ha sugerido que las fases iniciales de deformación pueden ser tan antiguas como el Oligoceno (26.6 a >24 Ma: Castillo-Reynoso, 2018) o pre-Mioceno tardío (Nieto-Obregón et al., 1981) con base en deformaciones observadas en campo en rocas de 16-14 Ma. Cabe señalar que el primer pulso de deformación reconocido por Castillo-Reynoso (2018) en la zona de cizalla del río Santiago es de carácter transpresional que, de prolongarse al oriente del área analizada por ese autor, también conduciría a la creación de relieve en el extremo meridional de la paleocuenca de Teocaltiche. La edad del inicio de actividad del rift Chapala-Tula se desconoce, ya que las fallas actuales solo se observan afectando a la secuencia volcánica del Mioceno tardío (ca. 11 - 5 Ma: Gómez-Tuena et al., 2005), que cubre toda el área junto con extensos rellenos sedimentarios de origen fluvio-lacustre.

En la Figura 10 se muestran aspectos diferentes de un modelo hipotético en el que el levantamiento tectónico en el bloque de piso de una falla normal importante causa el bloqueo parcial de un río axial que fluye adentro de un semigraben similar al de Teocaltiche. En el mismo diagrama se muestran áreas en donde la cuenca tectónica debió de experimentar subsidencia por la acumulación de los sedimentos (i.e. en y alrededor del depocentro de la cuenca) y otras regiones, coincidentes con áreas que aportaron los sedimentos, que debieron ascender debido al ajuste litostático causado por la erosión (Figura 10b). En estos diagramas, la falla $\sim \mathrm{E}-\mathrm{W}$ representaría ya sea el rift Tepic-Zacoalco o

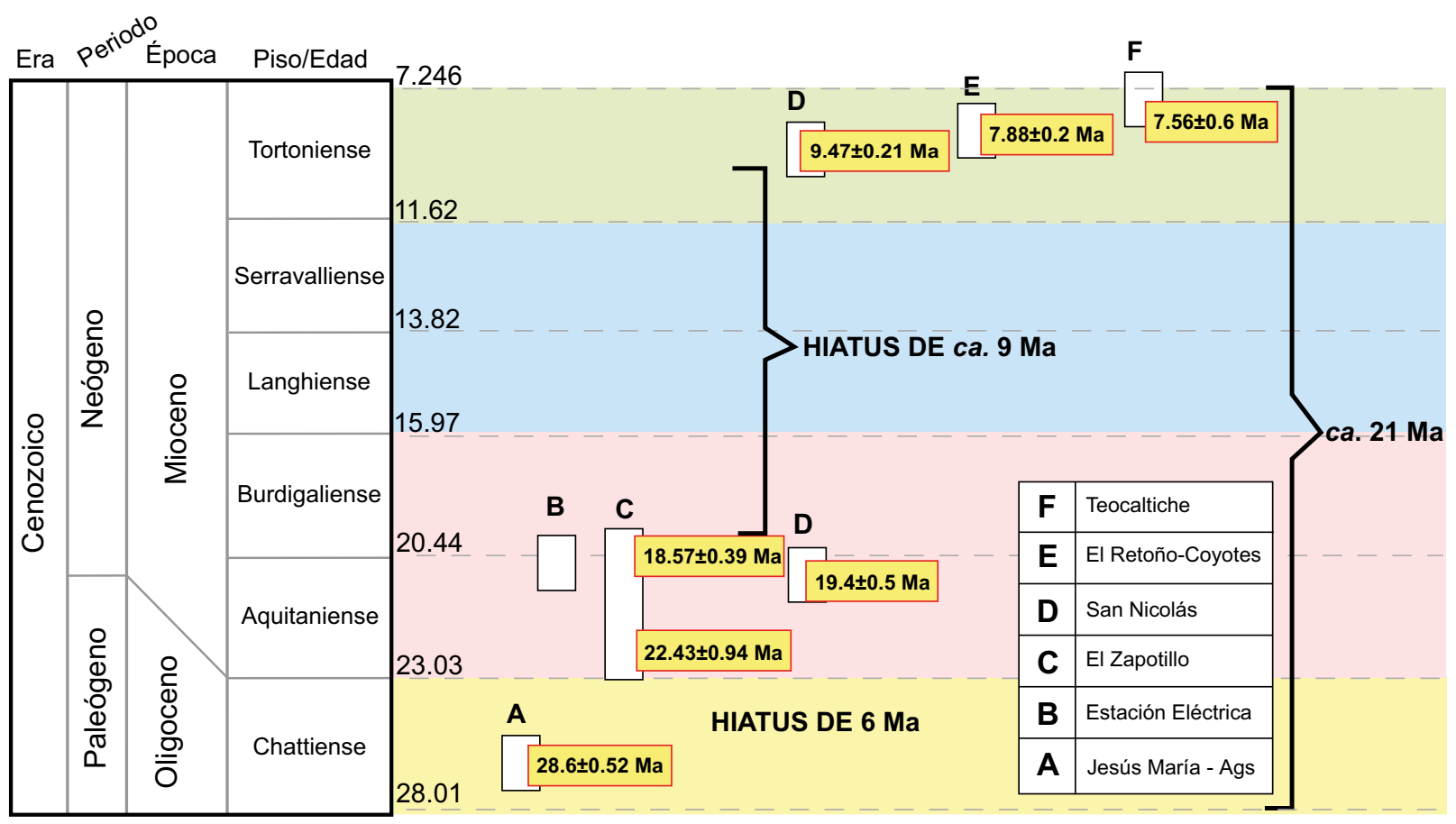

Figura 9. Tabla geocronológica en la que se graficaron las edades de las muestras fechadas de la paleocuenca de Teocaltiche. Note que la escala numérica no está dibujada a escala. 
el rift Chapala-Tula, dependiendo de la estructura burdamente $\sim \mathrm{N}-\mathrm{S}$ (Bolaños, Tlaltenango, Juchipila o Teocaltiche) del extremo meridional de la SMOc que se trate de explicar (Figura 10a y 10d). Cabe hacer notar que en al menos uno de los grábenes (Tlaltenango) de la parte sudoccidental de la SMOc el río axial fluye de sur a norte en su porción meridional (Montgomery y López-Blanco, 2003) y que estos autores discutieron la posibilidad de un levantamiento por ajuste isostático para explicar la evolución del drenaje en la región, aunque sin elucubrar acerca de la edad. En este mismo diagrama (Figura 10) se ilustra otra falla $\sim \mathrm{NE}-\mathrm{SW}$, la cual también genera subsidencia hacia el oeste, causando que el depocentro de la cuenca fluctuara entre el centro y oeste del área (Figura 10c); dicha falla representa la falla maestra del semigraben de Teocaltiche.

Las edades isotópicas obtenidas en los sedimentos indican sedimentación fluvio-lacustre durante el lapso entre $\sim 28$ y $\sim 7 \mathrm{Ma}$, pero no prueban que durante todo ese tiempo la depresión tectónica contuviera un solo lago perenne, ni tampoco aportan información detallada acerca de su extensión durante distintos lapsos de tiempo en que el tamaño y profundidad del lago pudieron variar, o incluso desaparecer temporalmente. El motivo de las posibles fluctuaciones en el nivel y extensión del lago están más allá de los alcances de este trabajo y serán discutidos en trabajos posteriores.

Finalmente, algunas preguntas que quedan por ser tratadas son ¿cuándo desaparecieron los paleolagos en las cuencas tectónicas del extremo meridional de la SMOc? y ¿cuál(es) fue(ron) el (los) proceso(s) que causó (aron) su desecación? En el área estudiada no se ha encontrado información relevante al respecto, ya que al sur de la presa El Zapotillo, afloran volcanes del Mioceno medio que cubren la secuencia sedimentaria-volcánica. El tema de la apertura e inicio de la erosión del relleno de las cuencas no ha sido discutido en la literatura, pero se hace notar que al igual que en el caso del bloqueo de las cuencas, si la apertura se hizo de manera sincrónica, se requiere de un mecanismo de carácter regional. El cambio climático conocido como el Evento Terminal del Mioceno (Chapin, 2008) causó el inicio de la desertificación de Norteamérica, con la consecuente disminución del escurrimiento, en el lapso entre 7 y $5 \mathrm{Ma}$ antes del presente tuvo un impacto considerable en la evolución de las redes de drenaje que fluían hacia el Pacífico (e.g., Aranda-Gómez et al., 2018). La disminución radical de la cantidad de escurrimiento potencialmente hubiera conducido a la transformación de las condiciones de lago abierto a las de un lago-playa, con precipitación de evaporitas, cosa que no se ha encontrado hasta ahora en la cuenca de Teocaltiche, ni en Juchipila o Tlaltenango. Por tanto, se cree que la apertura sucedió antes del arranque de la desertificación.

La formación del Golfo de California claramente causó un cambio sustancial en los perfiles de los ríos principales de la vertiente del Pacífico en el noroeste y centro de México, al redefinir drásticamente la distancia entre los parteaguas de las cuencas y el nivel base para la erosión (e.g., Aranda-Gómez et al., 2018). Aunque la primera formación de fondo marino cerca del golfo sucedió hace $3.5 \mathrm{Ma}$ (Castillo et al., 2002), hecho que señala la transferencia de la península de Baja California a la placa del Pacífico, se sabe que antes hubo una extensa depresión tectónica que fue invadida por el océano, ya que hay sedimentos marinos en Santa Rosalía (B.C.S.) con una edad isotópica de $7.1 \mathrm{Ma}$ (Holt et al., 2000). En la cuenca de Juchipila, a pesar de haberse prospectado por vertebrados fósiles en la región donde afloran sedimentos fluvio-lacustres por más de una década, no se han encontrado fósiles del Blancano ( $<4.75 \mathrm{Ma}$ ), lo que se interpreta como evidencia de que no hay sedimentos del Blancano y que la agradación había terminado a inicios del Plioceno. Aunque la actividad volcano-tectónica en los rifts Tula-Chapala y Tepic-Zacoalco no disminuyó después de la apertura del golfo, el levantamiento de los bloques de piso de las fallas continuó.
La erosión fluvial en los grábenes de la parte meridional de la SMOc debió incrementarse considerablemente por la incursión marina en la zona ahora ocupada por el Golfo de California, permitiendo la erosión parcial de las secuencias de relleno de graben en Bolaños, Juchipila y Teocaltiche. El único sitio en donde el levantamiento tectónico fue capaz de "derrotar" al río (river defeat), impidiendo su paso, fue en la cuenca de Tlaltenango, en donde el río aún fluye de sur a norte. En dicho graben no existe en la actualidad un lago debido a la captura del río Tlaltenango hecha por el río Bolaños (Montgomery y LópezBlanco, 2003).

\section{CONCLUSIONES}

En la región comprendida entre Aguascalientes, Teocaltiche y San Juan de los Lagos existió durante finales del Paleógeno y el Neógeno un lago. Edades isotópicas obtenidas en muestras de un afloramiento bien expuesto, en donde afloran sedimentos pelágicos, indican que el lago estuvo presente en el lapso entre $\sim 19$ y $9 \mathrm{Ma}$. Otras edades extienden el alcance temporal de la secuencia fluvio-lacustre hasta el rango entre $\sim 28$ y 7 Ma. Los rangos de edad se basan en análisis isotópicos $\mathrm{U}-\mathrm{Pb}$ en circones y en una edad biocronológica.

Una toba intercalada con arenas fluviales en la parte septentrional del área sugiere que el graben de Aguascalientes, que atrapó a la toba y sedimentos asociados, pudo comenzar su actividad poco después del inicio de la extensión en el área. La edad de formación del semigraben de Teocaltiche podría ser semejante a la del graben de Aguascalientes (> $28 \mathrm{Ma})$.

En la porción sudoriental de la SMOc hay cuatro cuencas de origen tectónico, relacionadas a extensión cortical. En al menos tres de ellas (Teocaltiche, Juchipila y Tlaltenango) hubo sedimentación fluviolacustre durante el Henfiliano. La existencia simultánea, al menos en parte, de tres lagos al sur de la SMOc requiere de una explicación de carácter regional para su formación y, posiblemente, también para su apertura. Un mecanismo viable para el cierre parcial simultáneo de las cuencas tectónicas y para la consecuente acumulación de sedimentos sería levantamiento en la región ubicada al norte del río Grande de Santiago. Una causa probable para el levantamiento regional sería el ajuste isostático de los bloques de piso de sistemas de fallas normales con rumbos $\sim$ E-W en la FVTM.

Para explicar la apertura simultánea de las tres cuencas lacustres el mecanismo podría relacionarse con la invasión marina en una depresión ubicada en donde ahora se encuentra el Golfo de California. Este cambio en el nivel base regional debió causar un aumento notable en el gradiente de los ríos axiales en los grábenes de la parte sudoriental de la SMOc, lo que dio a las corrientes fluviales la energía suficiente para remontar al levantamiento isostático aún activo, mediante erosión vigorosa, tanto en el lecho rocoso, como en los sedimentos de relleno de graben en las cuencas de origen tectónico.

\section{AGRADECIMIENTOS}

El apoyo económico para esta investigación fue proporcionado por el proyecto UNAM-DGAPA-PAPIIT IN100416 a J. Aranda. El mismo proyecto otorgó una beca parcial a N. González para realizar sus estudios de doctorado. Juan Tomás Vázquez elaboró láminas delgadas que se emplearon en la investigación. J. Jesús Silva Corona realizó la edición técnica del manuscrito y obtuvo la fotografía del fósil. Los comentarios de Dr. Raúl Miranda Avilés y de otros dos revisores externos ayudaron a mejorar notablemente el texto. Teresa Orozco realizó la edición final del texto. 

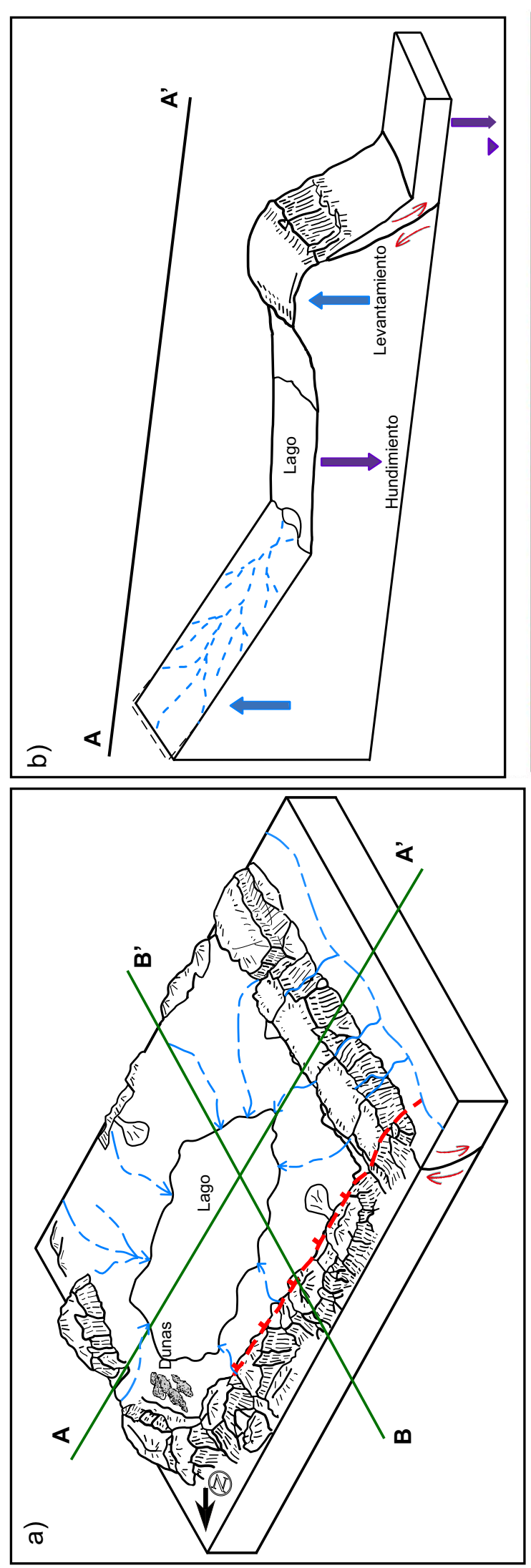
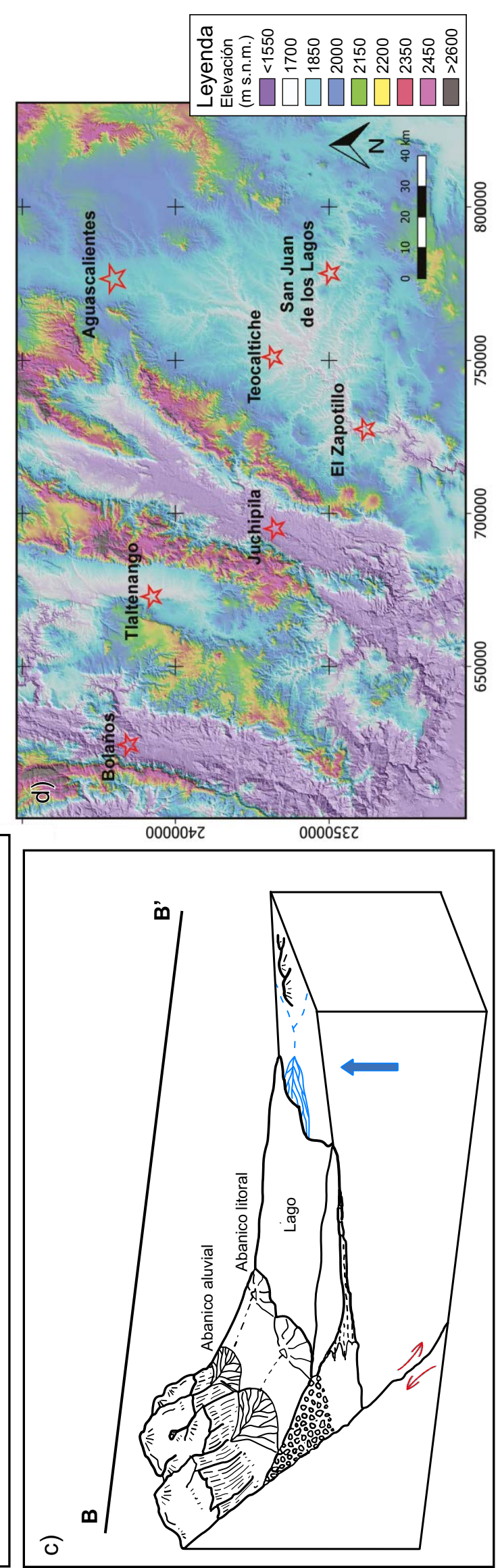

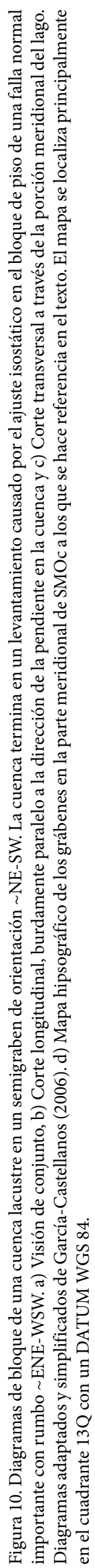




\section{MATERIAL SUPLEMENTARIO}

Los Anexos S1 y S2 pueden ser descargados del portal web de la revista $<$ http://rmcg.unam.mx/ $>$, en la sección de contenido de este número.

\section{REFERENCIAS}

Aguirre-Díaz, G., Carranza-Castañeda, O., 2000, Las grandes cuencas del OligoMioceno del centro de México, en Reunión anual de la Unión Geofísica Mexicana: Puerto Vallarta, México, Resumen p. 301.

Aranda-Gomez, J.J., 1989, Geología preliminar del graben de Aguascalientes: Revista Mexicana de Ciencias Geológicas, 8(1), 22-32.

Aranda-Gómez, J.J., Carranza-Castañeda, O., Wang, X., Tseng Z.J., Pacheco-Castro, A., 2018, Notes on the origin of extensive endorheic regions in central and northern Mexico, and some implications for paleozoogeography: Journal of South American Earth Sciences, 83, 55-67.

Banks, N.L., Bardwell K.A., Musiwa S., 1995, Karoo Rift basins of the Luangwa Valley, Zambia. Hydrocarbon Habitat in Rift Basins: Geological Society Special Publication, 80, 285-295.

Bertrand, S., Daga, R., Bedert, R., Fontijn, K., 2014, Deposition of the 2011-2012 Cordón Caulle tephra (Chile, $40^{\circ} \mathrm{S}$ ) in lake sediments: Implications for tephrochronology and volcanology: Journal of Geophysical ResearchEarth Surface, 119, 2555-2573, doi:10.1002/ 2014JF003321

Boygle, J.E., 1994, Tephra in lakes sediments: an unambiguous geochronological marker?: Edinburgh, UK, The University of Edinburgh, tesis de doctorado, $250 \mathrm{pp}$.

Boygle, J.E., 1999, Variability of tephra in lakes and catchment sediments, Svínavatn, Iceland: Global and Planetary Change, 21, 129-149.

Burbank, D.W., Anderson, R.S., 2001, Tectonic geomorphology: Malden MA, Blackwell Science, $274 \mathrm{pp}$.

Caravella, J.C., 1985, Lacustrine deposits in northeastern Yahualica de Gonzales Gallo quadrangle, Jalisco, Mexico. New Orleans LA, University of New Orleans, tesis de maestría, $118 \mathrm{pp}$.

Carranza-Castañeda, O., 2006a, Late Tertiary fossil localities in central Mexico, between $19^{\circ}-23^{\circ} \mathrm{N}$, en Carranza-Castañeda, O., Lindsay, E.H. (eds), Advances in late Tertiary vertebrate paleontology in Mexico and the Great American Biotic Interchange: Universidad Nacional Autónoma de México, Centro de Geociencias, Publicación especial 4, 45-60.

Carranza-Castañeda, O., Aranda-Gómez, J.J., Aguirre-Díaz, G., Nieto-Obregón, J., 2006b, Estratigrafía del Terciario tardío en la Cuenca de Juchipila-Jalpa, Estado de Zacatecas y sus implicaciones para el gran intercambio de faunas entre las Américas, Geos, 26 (1), 132.

Carranza-Castañeda, O., Aranda-Gómez, J.J., Wang, X., 2013, The earlylate Hemphillian (Hh2) faunal assemblage form Juchipila basin, state of Zacatecas, Mexico, and its biochronologic correlation with other Hemphillian faunas in Central Mexico: Contributions in Science, 521, 13-49.

Castillo, P.R., Hawkins, J.W., Lonsdale, P.F., Hilton, D.R., Shaw, A.M., 2002, Petrology of Alarcon Rise lavas, Gulf of California: Nascent intracontinental ocean crust: Journal of Geophysical Research, 107 (B10), p. doi:10.1029/20011JB000666

Castillo-Reynoso, J.C., 2018, Tectónica del límite sur de la Sierra Madre Occidental: la zona de cizalla del Río Santiago: Universidad Nacional Autónoma de México, Posgrado en Ciencias de la Tierra, tesis de maestría, $174 \mathrm{pp}$.

Chapin, C.E., 2008, Interplay of oceanographic and paleoclimate events with tectonism during the middle to late Miocene sedimentation across the southwestern USA: Geosphere, 4(6), 976-991.

De Cserna, Z., Álvarez, R., 1995, Quaternary drainage development in Central Mexico and the threat of an environmental disaster: a geological appraisal: Environmental \& Engineering Geoscience 1(1), 737, 29-34.

Delgado-Argote, L., Nieto-Obregón J., Damon P.E., 1981, Evolución magmática de la junta de la Sierra Madre Occidental y el Eje Neovolcánico, en 77th Annual meeting of the Geological Society of America, Cordilleran Section: CIUDAD, PAÍS, Geological Society of America, Abstracts with programs, 13(2), 52 .

Doeglas, D.J., 1968, Grain-size indices, classification and environment:
Sedimentology, 10, 83-100.

Dugés, A., 1894, Felis fósil de San Juan de los Lagos: La Naturaleza, 2, 412-423. Ferrari, L., Orozco-Esquivel, T., Manea, V., Manea, M., 2012, The dynamic history of the Trans-Mexican Volcanic Belt and the Mexico subduction zone: Tectonophysics, 522-523, 122-149.

Folk, R.L., 1969, Petrology of sedimentary rocks: Austin TX, Hemphill Publishing Company, $184 \mathrm{pp}$.

Gámez-Vintaned, J.A., Liñá, P.E., 1996, Revisión de la terminología icnológica en español: Revista Española de Paleontología, 11(2), 155-176. ISSN 0213-6937.

García-Castellanos, D., 2006, Long-term evolution of tectonic lakes: Climatic controls on the development of internally drained basins, en Willett, S.D., Hovius, N., Brandon, M.T., Fisher, D.M., (eds), Tectonics, Climate, and Landscape Evolution: Geological Society of America Special Paper 398, 283-294, doi: 10.1130/2006.2398(17)

Goddard, E.N., Trask, P.D., DeFord, R.K., Rove, O.N., Jr. Singewald, J.T., Overbeck, R.M. 1963, Rock-color Chart: Boulder CO, The Geological Society of America, 16 pp.

Gómez-Tuena, A., Orozco-Esquivel, M.T., Ferrari, L., 2005, Petrogénesis ígnea de la Faja Volcánica Transmexicana: Boletín de la Sociedad Geológica Mexicana, LVII(3), 227-285.

Gray, J.E., 1821, On the natural arrangement of vertebrose animals: London Medical Repository 15: 296-310

Guzmán-Gutiérrez, R., Rodríguez-Huerta, M., 1991, Un nuevo registro de Osteoborus (Canidae, Carnivora) para México, en 3rd Congreso Nacional de Paleontología: México D.F., Sociedad Mexicana de Paleontología, Resúmenes, 108-109.

Hernández-Láscares, D., 1981, Estratigrafía de la Región Central de Aguascalientes, Ags., México, Gaceta Geológica de México, 31, 17-40.

Holt, J.W., Holt, E.W., Stock, J.M., 2000, An age constraint on the Gulf of California rifting from the Santa Rosalía basin, Baja California Sur, Mexico: Geological Society of America Bulletin, 112(4), 540-549.

INEGI (Instituto Nacional de Estadística, Geografía e Informática), 1971a, Carta geológica Jalostotitlán F-13-D-48, escala 1:50,000: México. D.F., Secretaría de Programación y Presupuesto, Instituto Nacional de Estadística, Geografía e Informática, 1 mapa.

INEGI (Instituto Nacional de Estadística, Geografía e Informática), 1971b, Carta geológica San Juan de los Lagos F-13-D-49, escala 1:50,000: México. D.F., Secretaría de Programación y Presupuesto, Instituto Nacional de Estadística, Geografía e Informática, 1 mapa.

INEGI (Instituto Nacional de Estadística, Geografía e Informática), 1973a, Carta geológica Encarnación de Díaz F-13-D-29, escala 1:50,000: México. D.F., Secretaría de Programación y Presupuesto, Instituto Nacional de Estadística, Geografía e Informática, 1 mapa.

INEGI (Instituto Nacional de Estadística, Geografía e Informática), 1973b, Carta geológica Jesús María F-13-D-18, escala 1:50,000: México. D.F., Secretaría de Programación y Presupuesto, Instituto Nacional de Estadística, Geografía e Informática, 1 mapa.

INEGI (Instituto Nacional de Estadística, Geografía e Informática), 1973c, Carta geológica Villa Hidalgo F-13-D-28, escala 1:50,000: México. D.F., Secretaría de Programación y Presupuesto, Instituto Nacional de Estadística, Geografía e Informática, 1 mapa.

INEGI (Instituto Nacional de Estadística, Geografía e Informática), 1973d, Carta geológica Yahualica de González Gallo F-13-D-47, escala 1:50,000: México. D.F., Secretaría de Programación y Presupuesto, Instituto Nacional de Estadística, Geografía e Informática, 1 mapa.

INEGI (Instituto Nacional de Estadística, Geografía e Informática), 1977, Carta geológica Aguascalientes F-13-D-19, escala 1:50,000: México. D.F., Secretaría de Programación y Presupuesto, Instituto Nacional de Estadística, Geografía e Informática, 1 mapa.

INEGI (Instituto Nacional de Estadística, Geografía e Informática), 1980, Carta geológica Teocaltiche F-13-D-38, escala 1:50,000: México. D.F., Secretaría de Programación y Presupuesto, Instituto Nacional de Estadística, Geografía e Informática, 1 mapa.

INEGI (Instituto Nacional de Estadística, Geografía e Informática), 1981, Carta geológica Santa Bárbara F-13-D-39, escala 1:50,000: México. D.F., Secretaría de Programación y Presupuesto, Instituto Nacional de Estadística, Geografía e Informática, 1 mapa.

INEGI (Instituto Nacional de Estadística, Geografía e Informática), 2010, 
Simulador de flujos de agua de cuencas hidrográficas (SIATL), disponible en <http://antares.inegi.org.mx/analisis/red_hidro/siatl/>

IMTA (Instituto Mexicano de Tecnología del Agua), 2007, Elaboración de los documentos de respaldo de la disponibilidad media anual de agua en los acuíferos (1413) Altos de Jalisco, (1436) Arenal, (1428) 825 Chapala, (1429) Tizapán y (1447) Yahualica, en el Estado de Jalisco. Acuífero (1447) Yahualica, Informe final, Convenio de colaboración CNA-OCLSP-JALCONV-004-2007: México, D.F., inédito.

Johnson, C.A., Harrison, C.G.A., 1990, Neotectonics in central Mexico: Physics of the Earth and Planetary Interiors, 64, 187-210.

Keller E.A., Pinter, N., 1996, Active tectonics: earthquakes, uplift and landscape: Upper Saddle River NJ, Prentice Hall, 362 pp.

Lahiere, L., 1982, Petrology of lacustrine deposits. Juchipila quadrangle, Zacatecas, México: New Orleans LA, University of New Orleans, tesis de maestría. $95 \mathrm{pp}$.

Leeder M.R., Jackson J.A., 1993, The interaction between normal faulting and drainage in active extensional basins, with examples from the western United States and central Greece: Basin Research, 5, 79-102.

López. J.C. 1991, Stratigraphy and petrology of Miocene-Pliocene lacustrine deposits, Juchipila, Quadrangle. Zacatecas, México: New Orleans LA, University of New Orleans, tesis de maestría, $217 \mathrm{pp}$.

Loza-Aguirre, I., Nieto-Samaniego, A.F., Alaniz-Alvarez, S.A., Iriondo, A., 2008, Relaciones estratigráfico-estructurales en la intersección del sistema de fallas San Luis-Tepehuanes y el graben de Aguascalientes, México Central: Revista Mexicana de Ciencias Geológicas, 25(3), 533-548.

Matthew, W.D., Stirton, R.A., 1930, Equidae from the Pliocene of Texas, University of California Publications: Bulletin of Geological Sciences, 19, 349-396.

McDowell, F.W., Keizer, R.P., 1977, Timing of mid-Tertiary volcanism in the Sierra Madre Occidental between Durango City and Mazatlan, Mexico: Geological Society of America Bulletin, 88, 1479-1486.

Montellano-Ballesteros, M., 1989, Pliocene camelids from the Rancho El Ocote, Guanajuato, México: Journal of Mammalogy, 70, 2, 358-368.

Montellano-Ballesteros, M., 1990 (1992), Una edad del Irvingtoniano al Rancholabreano para la fauna Cedazo del Estado de Aguascalientes: Universidad Nacional Autónoma de México, Revista del Instituto de Geología, 9(2), 195-203.

Montellano-Ballesteros, M., 1997, New vertebrate locality of late Hemphillian age in Teocaltiche, Jalisco, Mexico: Revista Mexicana de Ciencias Geológicas, 14(1), 84-90.

Montgomery, D.R., López-Blanco, J., 2003, Post-Oligocene river incision, southern Sierra Madre Occidental, Mexico: Geomorphology, 55, 235-247.

Mooser, O., 1958, La fauna "Cedazo" del Pleistoceno de Aguascalientes: Universidad Nacional Autónoma de México, Anales del Instituto de Biología 29, 408-452.

Mooser, O., Dalquest, W.W., 1975, Pleistocene mammals from Aguascalientes, Central México: Journal of Mammalogy, 56, 781-820.

Nieto-Obregon, J., Delgado-Argote, L., Damon, P.E., 1981, Relaciones petrológicas y geocronológicas del magmatismo de la Sierra Madre Occidental y el Eje Neovolcanico en Nayarit, Jalisco y Zacatecas: Asociación de Ingenieros Mineros, Metalurgistas y Geólogos de México, Memoria Técnica 14, 327-361.

Nieto-Samaniego, A.F., Macías. Romo, C., Alaniz-Alvarez, S.A., 1996, Nuevas edades isotópicas de la cubierta volcánica cenozoica de la parte meridional de la Mesa central, México: Revista Mexicana de Ciencias Geológicas, 13(1), 117-122.

Nieto-Samaniego, Á.F., Ferrari, L., Alaniz-Álvarez, S.A., Labarthe-Hernández, G., Rosas-Elguera, J., 1999, Variation of Cenozoic extension and volcanism across the southern Sierra Madre Occidental volcanic province, Mexico: Geological Society of America Bulletin, 111, 347-363.

Norma Oficial Mexicana NOM-021-RECNAT-2000, 2002, Que establece las especificaciones de fertilidad, salinidad y clasificación de suelos. Estudios y análisis: México, Diario Oficial de la Federación, martes 31 de diciembre de 2002, 73 pp.

Olson, E.E., McGrew, P.O., 1941, Mammalian fauna from the Pliocene of Honduras: Bulletin of the Geological Society of America, 52, 1219-1244.
Ortega-Gutiérrez, F., Mitre-Salazar, L.M., Roldán-Quintana, J., Aranda-Gómez J.J., Morán-Zenteno, D., Alaníz-Alvarez S.A., Nieto-Samaniego, A.F., 1992, Carta geológica de la República Mexicana: México, Universidad Nacional Autónoma de México, Instituto de Geología, Secretaría de Energía, Minas e Industria Paraestatal y Consejo de Recursos Minerales, 1 mapa y texto explicativo).

Ortega-Obregón, C., Solari, L., Gómez-Tuena, A., Elías-Herrera, M., OrtegaGutiérrez, F., Macías- Romo, C., 2014, Permian-Carboniferous arc magmatism in southern Mexico: U-Pb dating, trace element and $\mathrm{Hf}$ isotopic evidence on zircons of earliest subduction beneath the western margin of Gondwana: International Journal of Earth Sciences, 103(5), 1287-1300.

Owen, R., 1848, Description of teeth and portion of jaws of two extinct anthracotherioid qudrupeds discovery by the Marchioness of Hastings in the Eocene deposits of the Island of Wright: Geological Society of London Quarterly Journal, 4, 103-145.

Platt N. H., Wright V. P, 1991, Lacustrine carbonates: facies models, facies distributions and hydrocarbon aspects, en Anadón, P., Cabrera, LI., Kelts, K. (eds.), Lacustrine Facies Analysis: International Association of Sedimentologists Special Publication 13 13, 57-74.

Rodríguez-de la Rosa, R.A., Guzmán-Gutiérrez, J.R., 2012, Huellas de aves y mamíferos del Neógeno de Jalisco, México: Paleontología Mexicana, $62,147-158$

Sánchez-González, A., Vázquez-Tortoledo, R., Romo-Ramírez, J., de la CallejaMoctezuma, A., Guerrero-Armenta, A.A., 1998, Carta geológico-minera Aguascalientes F13-9, esc. 1:250,000, Ags., Jal., Zac. y Nay: Pachuca, Hgo., Servicio Geológico Mexicano, 1 mapa.

Scheubel, F.R., Clark, K.F., Porter, E.W., 1988, Geology, tectonic environment, and structural controls in the San Martin de Bolaños District, Jalisco, Mexico: Economic Geology, 83, 1703-1720.

Schnurrenberger, D., Russell J., Kelts, K., 2003, Classification of lacustrine sediments based on sedimentary components: Journal of Paleolimnology, 29(2), 141-154.

SGM (Servicio Geológico Mexicano), 1998, Carta geológico-minera Aguascalientes F13-9, escala 1:250,000: Pachuca, Hidalgo, México, Servicio Geológico Mexicano, 1 mapa.

Solari, L.A., Gómez-Tuena, A., Bernal, J.P., Pérez-Arvizu, O., Tanner, M., 2010, $\mathrm{U}-\mathrm{Pb}$ zircón geochronology by an integrated LA-ICPMS microanalytical workstation: achievements in precisión and accuracy, Geostandards and Geoanalytical Research, 34(1), 5-18.

Stow, D.A.V., 2005, Sedimentary Rocks in the field: a colour guide: Boca Raton FL, CRC Press, 320pp.

Tedford, R.H., Albright, L. B.III, Barnosky, A.D., Ferrusquia-Villafranca, I., Hunt, R. M. Jr., Storer, J.E., Swisher, C.C.III, Voorhies, M.R., Webb, S.D., Whistler, D. P., 2004, Mammalian biochronology of the Arikareean through Hemphillian interval (late Oligocene through earliest Pliocene epochs), en Woodburne, M.O. (ed.), Late Cretaceous and Cenozoic Mammals of North America, Biostratigraphy and Biochronology: New York NY, Columbia University Press, 169-231.

Tristán-González M., Aguirre-Díaz G.J., Labarthe-Hernández G., TorresHernández J.R., Bellon H., 2009, Post-Laramide and pre-Basin and Range deformation and implications for Paleogene (55-25Ma) volcanism in central Mexico: A geological basis for a volcano-tectonic stress model: Tectonophysics, 471, 136-152.

UAQ-UNAM-UAA, 2002, Estudio integral sobre la problemática del agua, subsidencia y sismicidad en el Valle de Aguascalientes: Aguascalientes, México: Facultad de Ingeniería de la UAQ, Centro de Geociencias de la UNAM, Instituto de Geofísica de la UNAM y Centro del Diseño y la Construcción UAA, Instituto del Agua de Aguascalientes, Informe técnico $433 \mathrm{pp}$.

Wentworth, C.K., 1922, A scale of grade and class terms for clastic sediments: The Journal of Geology, 30, 377-392.

Manuscrito recibido: abril 10, 2019

Manuscrito corregido recibido: agosto 18, 2019

Manuscrito aceptado: agosto 21, 2019 